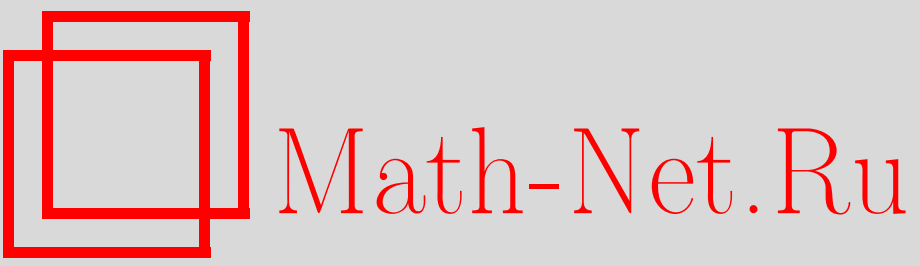

М. Е. Палистрант, Сверхпроводимость и ферромагнетизм в неадиабатических системах с магнитной примесью. Выход за рамки теоремы Мигдала, ТМФ, 2003, том 135, номер 1, 137-158

DOI: https://doi.org/10.4213/tmf177

Использование Общероссийского математического портала Math-Net.Ru подразумевает, что вы прочитали и согласны с пользовательским соглашением

http: //www . mathnet.ru/rus/agreement

Параметры загрузки:

IP : 18.234 .156 .22

26 апреля 2023 г., 13:56:43 
ТЕОРЕТИЧЕСКАЯ

И МАТЕМАТИЧЕСКАЯ

ФИЗИКА

Том 135, № 1

апрель, 2003

(C) 2003 г.

М.Е. Палистрант ${ }^{*}$

\title{
СВЕРХПРОВОДИМОСТЬ И ФЕРРОМАГНЕТИЗМ В НЕАДИАБАТИЧЕСКИХ СИСТЕМАХ С МАГНИТНОЙ ПРИМЕСЬЮ. ВЫХОД ЗА РАМКИ ТЕОРЕМЫ МИГДАЛА
}

\begin{abstract}
Изучена возможность ферромагнитного упорядочения парамагнитной примеси в неадиабатических сверхпроводящих системах. Учитываются эффект "раздвижки" поверхности Ферми внутренним магнитным полем, обменное взаимодействие ионов примеси через электроны проводимости и спин-орбитальное взаимодействие немагнитной примеси. Задача решается в линейном по неадиабатичности приближении путем учета вершинных и пересекающихся диаграмм по электрон-фононному и электрон-примесному взаимодействию. Получены основные уравнения теории сверхпроводимости неадиабатических систем с ферромагнитным упорядочением спинов примеси, и выявлено влияние неадиабатичности на температуру сверхпроводящего перехода $T_{\mathrm{c}}$ и на критическую концентрацию примеси. Изучено также поведение температуры магнитного упорядочения $T_{\mathrm{K}}$ как функции от концентрации примеси $c$ в сверхпроводящем состоянии неадиабатической системы. Построена фазовая диаграмма $(T, c)$, и показано, что эффекты неадиабатичности способствуют увеличению области сосуществования сверхпроводимости и ферромагнетизма.
\end{abstract}

Ключевые слова: сверхпроводимость, ферромагнетизм, примесь, неадиабатичность.

\section{1. ВВЕДЕНИЕ}

За годы, прошедшие после открытия высокотемпературной сверхпроводимости (ВТСП), достигнуты значительные успехи как в экспериментальных, так и в теоретических исследованиях этого эффекта. Однако механизм возникновения ВТСП пока не выявлен. Следовательно, при теоретических исследованиях наряду с рассмотрением различных "экзотических" моделей остается актуальным подход, в основе которого лежит электрон-фононное взаимодействие. Это взаимодействие, в частности, определяет сверхпроводимость в фуллеренах [1], в соединениях $\mathrm{MgB}_{2}\left(T_{\mathrm{c}}=40 \mathrm{~K}\right)$ [2], наблюдается также сильное влияние электрон-фононного взаимодействия на динамику электронов в оксидных керамиках [3].

* Институт прикладной физики, Кишинёв, Молдавия. E-mail: statphys@asm.md 
Даже если этот механизм не является основным в некоторых материалах, несомненно, он играет значительную роль. В связи с этим по-прежнему важное значение имеют идеи и методы, изложенные в классических работах [4]-[11] для пространственно однородных систем и в работах [12]-[17] для пространственно неоднородных. Дальнейшее развитие теории сверхпроводимости на базе фононного механизма применительно к ВТСП-материалам кратко изложено, например, в монографии [18] и работе [19]. Важную роль в получении высоких температур сверхпроводящего перехода $T_{\mathrm{c}}$ играют сильная электрон-фононная связь [20], [21], ангармонический характер колебаний ионов решетки [22], [23], наличие особенности Ван Хова или плоских участков в электронном спектре [24], [25], перекрытие энергетических зон на поверхности Ферми [26], [27].

Наряду с этим имеется еще одна важная особенность оксидных керамик, фуллеренов и органических сверхпроводников: они являются неадиабатическими системами. В этих системах энергия Ферми $\left(\varepsilon_{\mathrm{F}}\right)$ и энергия Дебая $\left(\omega_{\mathrm{D}}\right)$ являются величинами одного порядка. Это обстоятельство приводит к нарушению теоремы Мигдала [9], которая выполняется в обычных сверхпроводниках $\left(\varepsilon_{\mathrm{F}} \gg \omega_{\mathrm{D}}\right)$, и к необходимости учета дополнительных многочастичных эффектов. Математически это соответствует учету так называемых вериинных поправок. Это обстоятельство толкает на пересмотр теории сверхпроводимости как для чистых систем, так и для систем с примесью замешения.

Проблеме учета неадиабатичности посвящено много исследований, развиты различные методы, анализ которых приведен, например, в работе [28]. В частности, показано, что правильную оценку неадиабатических поправок собственной энергии дает метод прямого вычисления (аналогичный применявшемуся Мигдалом). Этот метод был использован в работах [29], [30], где с использованием гамильтониана фрелиховского типа получены уравнения Элиашберга для неадиабатических систем. При этом в определении массовых операторов $\Sigma_{N}$ и $\Sigma_{S}$ учтены линейные по неадиабатичности члены (диаграммы с пересечением только двух линий электрон-фононного взаимодействия).

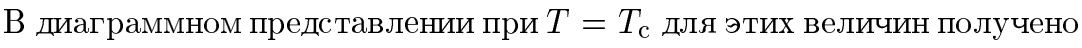

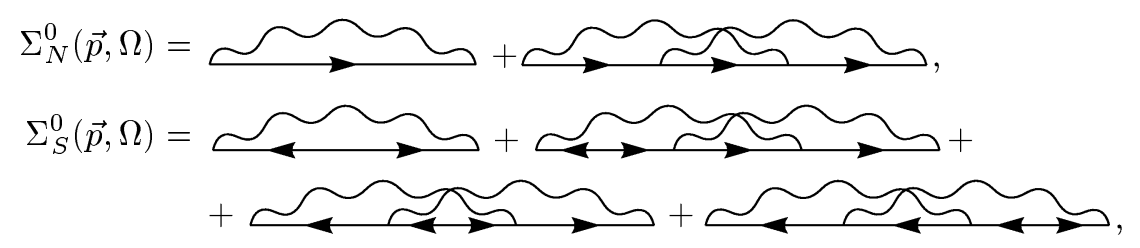

Здесь прямые линии со стрелками отвечают полной электронной функции Грина, волнистые - фононной. Эта схема соответствует разложению по параметру $\lambda \omega_{\mathrm{D}} / \varepsilon_{\mathrm{F}}$ и включает вклады первого порядка вне рамок теоремы Мигдала.

Вычисленные в работах [29], [30] вершинные и пересекаюшиеся функции содержат сложную зависимость от импульса обмена $q$ и частоты $\omega$. В частности, они положительны при малых $q\left(q \ll 2 p_{\mathrm{F}}\right)$. При этом происходит существенная перенормировка константы связи $\lambda$ в сторону ее увеличения, что, в свою очередь, приводит к повышению температуры сверхпроводяшего перехода $T_{\mathrm{c}}$. В результате оказывается возможным достижение значений $T_{\mathrm{c}}$, характерных для ВТСП-материалов, при промежуточных зна- 
чениях константы связи $(\lambda \sim 0.5-1.0)$. Отметим, что в обычном уравнении Элиашберга такие значения $T_{\mathrm{c}}$ соответствуют очень сильной связи $(\lambda \sim 3)$.

Малые же значения импульса обмена $q$, благоприятные для сверхпроводящего состояния, авторы работ [29], [30] связывают с наличием в системе сильных электронных корреляший, наличие которых приводит к слабому росту электрон-фононного взаимодействия в области малых $q$ и резкому убыванию его с ростом величины $q$ [31], [32]. Из этих результатов следует, что факторы, нарушаюшие теорему Мигдала (малые плотности носителей заряда и сильные электронные корреляции), могут служить серьезной основой для возникновения ВТСП.

Поскольку эффекты неадиабатичности столь сушественны, представляет несомненный теоретический и практический интерес изучение влияния неадиабатичности на сверхпроводящие свойства примесных систем.

В обычных сверхпроводниках парамагнитная примесь подавляет сверхпроводимость в результате распаривания куперовских пар из-за рассеяния электронов на магнитной примеси $[15],[16]$. При этом в области низких температур возможно сосушествование сверхпроводимости и ферромагнитного упорядочения спинов атомов примеси [17]. Это упорядочение происходит благодаря косвенному обменному взаимодействию ионов через электроны проводимости и вызывает появление пространственной плотности электронного спина, определяемой величиной парамагнитной восприимчивости, которая отлична от нуля у сверхпроводников даже при $T=0$ за счет эффектов обменного рассеяния электронов на парамагнитной примеси. Эти эффекты, а также спин-орбитальное взаимодействие противостоят "раздвижке" поверхности Ферми внутренним полем и приводят к возникновению смешанной фазы (сверхпроводимость и ферромагнетизм).

Целью данной работы является исследование влияния эффектов неадиабатичности на зависимости температуры сверхпроводяшего перехода $T_{\mathrm{c}}$ и температуры ферромагнитного упорядочения примеси $T_{\mathrm{K}}$ от концентрации хаотически распределенной магнитной примеси и, следовательно, на сосушествование сверхпроводимости и ферромагнетизма.

\section{2. ГАМИЛЬТОНИАН СИСТЕМЫ И ФУНКЦИИ ГРИНА}

Гамильтониан рассматриваемой системы представим в виде

$$
H=H_{0}+H_{1}+H_{2},
$$

где $H_{0}$ - гамильтониан свободных электронов и фононов, а $H_{1}$ и $H_{2}$ - гамильтонианы электрон-фононного и электрон-примесного взаимодействия, соответственно. Имеем

$$
\begin{aligned}
& H_{1}=\sum_{\alpha} \int d \vec{x} \psi_{\alpha}^{+}(\vec{x}) \psi_{\alpha}(\vec{x}) \varphi(\vec{x}), \\
& H_{2}=\sum_{\alpha \beta} \int d \vec{x} \psi_{\alpha}^{+}(\vec{x}) V_{\alpha \beta}(\vec{x}) \psi_{\beta}(\vec{x}), \quad V_{\alpha \beta}(\vec{x})=V_{1}(\vec{x}) \delta_{\alpha \beta}+V_{2}(\vec{x})(\vec{S} \vec{\sigma})_{\alpha \beta},
\end{aligned}
$$


$\psi_{\alpha}^{+}(\vec{x})$ и $\psi_{\alpha}(\vec{x})$ - операторы рождения и уничтожения электрона со спином $\alpha$ в точке $\vec{x}$, $\varphi(\vec{x})$ - фононный оператор, $\vec{S}$ - классический спин примесного атома, $\vec{\sigma}-$ спин-матричньй вектор, $V_{1}$ и $V_{2}$ - немагнитная и магнитная части потенциала рассеяния электронов на магнитной примеси.

Как известно, сверхпроводящая электрон-фононная система может быть описана температурными функциями Грина

$$
\begin{aligned}
G_{\alpha \beta}\left(x, x^{\prime}\right) & =-\left\langle T \psi_{\alpha}(x) \psi_{\beta}^{+}\left(x^{\prime}\right)\right\rangle, \\
F_{\alpha \beta}^{+}\left(x, x^{\prime}\right) & =-\left\langle T \psi_{\alpha}^{+}(x) \psi_{\beta}^{+}\left(x^{\prime}\right)\right\rangle, \\
F_{\alpha \beta}\left(x, x^{\prime}\right) & =-\left\langle T \psi_{\alpha}(x) \psi_{\beta}\left(x^{\prime}\right)\right\rangle, \\
D\left(x, x^{\prime}\right) & =-\left\langle T \varphi(x) \varphi\left(x^{\prime}\right)\right\rangle,
\end{aligned}
$$

где $x=(\vec{x}, \tau)$, а $\psi_{\alpha}(x), \varphi(x)$ - операторы в представлении Гайзенберга, $\langle\cdot\rangle-$ усреднение по состоянию системы взаимодействующих частиц.

Чтобы изучить сверхпроводящие свойства рассматриваемой системы, необходимо получить уравнения для функций Грина (6) с учетом электрон-фононного и электрон-примесного взаимодействий. С этой целью перейдем к представлению взаимодействия и запишем ряд теории возмушений для этих функций по взаимодействию $H_{1}+H_{2}$, применяя методику, описанную в книге [33]. Выполним затем усреднение по положениям хаотически распределенной примеси и ориентациям спинов. При этом имеем

$$
\begin{aligned}
\overline{V_{\alpha_{1} \beta_{1}}\left(\vec{x}_{1}\right) V_{\alpha_{2} \beta_{2}}\left(\vec{x}_{2}\right)}= & \frac{c}{V} \sum_{\vec{q}} e^{-i \vec{q}\left(\vec{x}_{1}-\vec{x}_{2}\right)}\left\{V_{1}(\vec{q}) V_{1}(-\vec{q}) \delta_{\alpha_{1} \beta_{1}} \delta_{\alpha_{2} \beta_{2}}+\right. \\
& +\left\langle S_{z}\right\rangle\left[V_{1}(\vec{q}) V_{2}(\vec{q}) \delta_{\alpha_{1} \beta_{1}} \sigma_{\alpha_{2} \beta_{2}}^{z}+V_{2}(\vec{q}) V_{1}(-\vec{q}) \delta_{\alpha_{2} \beta_{2}} \sigma_{\alpha_{1} \beta_{1}}^{z}\right]+ \\
& +V_{2}(\vec{q}) V_{2}(-\vec{q})\left[\left\langle S_{x}^{2}\right\rangle \sigma_{\alpha_{1} \beta_{1}}^{x} \sigma_{\alpha_{2} \beta_{2}}^{x}+\left\langle S_{y}^{2}\right\rangle \sigma_{\alpha_{1} \beta_{1}}^{y} \sigma_{\alpha_{2} \beta_{2}}^{y}+\right. \\
& \left.\left.+\left\langle S_{z}^{2}\right\rangle \sigma_{\alpha_{1} \beta_{1}}^{z} \sigma_{\alpha_{2} \beta_{2}}^{z}\right]\right\}
\end{aligned}
$$

Здесь и ниже черта сверху означает усреднение по хаотически распределенной примеси и ориентациям спинов, $c$ - концентрация примеси, $V_{1}(\vec{q})$ и $V_{2}(\vec{q})$ - фурье-компоненты потенциалов примесного рассеяния электронов.

В полученном ряде теории возмушений учитываем все диаграммы, характерные для примесных адиабатических систем, и дополнительные диаграммы, содержащие пересечения линий электрон-фононного и электрон-примесного взаимодействий. Эти дополнительные диаграммы и определяют вклад от неадиабатичности. Формула (7) содержит возможность ферромагнитного упорядочения $\left(\left\langle S_{z}\right\rangle \neq 0\right)$ спинов примеси. После суммирования вьшеуказанного ряда теории возмушений по электронным спиновым переменным получаем систему уравнений для функций Грина. $\mathrm{B}(\vec{k}, \Omega)$-представлении эта система имеет вид

$$
\begin{aligned}
\bar{G}(\vec{k}, \Omega)= & G^{0}(\vec{k}, \Omega)+G^{0}(\vec{k}, \Omega) \Sigma_{\alpha \alpha}^{\prime N}(\vec{k}, \Omega) \bar{G}_{\alpha \alpha}(\vec{k}, \Omega)- \\
& -G^{0}(\vec{k}, \Omega) \Sigma_{\alpha-\alpha}^{S}(\vec{k}, \Omega) \bar{F}_{-\alpha \alpha}^{+}(\vec{k}, \Omega), \\
\bar{F}_{-\alpha \alpha}^{+}(\vec{k}, \Omega)= & G^{0}(-\vec{k},-\Omega) \Sigma_{-\alpha-\alpha}^{\prime N}(-\vec{k},-\Omega) \bar{F}_{-\alpha \alpha}^{+}(\vec{k}, \Omega)+ \\
& +G_{0}(-\vec{k},-\Omega) \Sigma_{-\alpha \alpha}^{S+}(\vec{k}, \Omega) \bar{G}_{\alpha \alpha}(\vec{k}, \Omega),
\end{aligned}
$$


где $\alpha=\uparrow, \downarrow, \Sigma_{\alpha \alpha}^{\prime N}(\vec{k}, \Omega)=I \sigma_{\alpha \alpha}^{z}+\Sigma_{\alpha \alpha}^{N}(\vec{k}, \Omega), I=c V_{2}(0)\left\langle S_{z}\right\rangle$ - внутреннее поле. Массовые операторы $\Sigma_{\alpha \alpha}^{N}(\vec{k}, \Omega)$ и $\Sigma_{\alpha-\alpha}^{S}(\vec{k}, \Omega)$ включают в себя все порядки теории возмушений адиабатической теории и первый порядок по неадиабатичности.

На языке диаграмм имеем

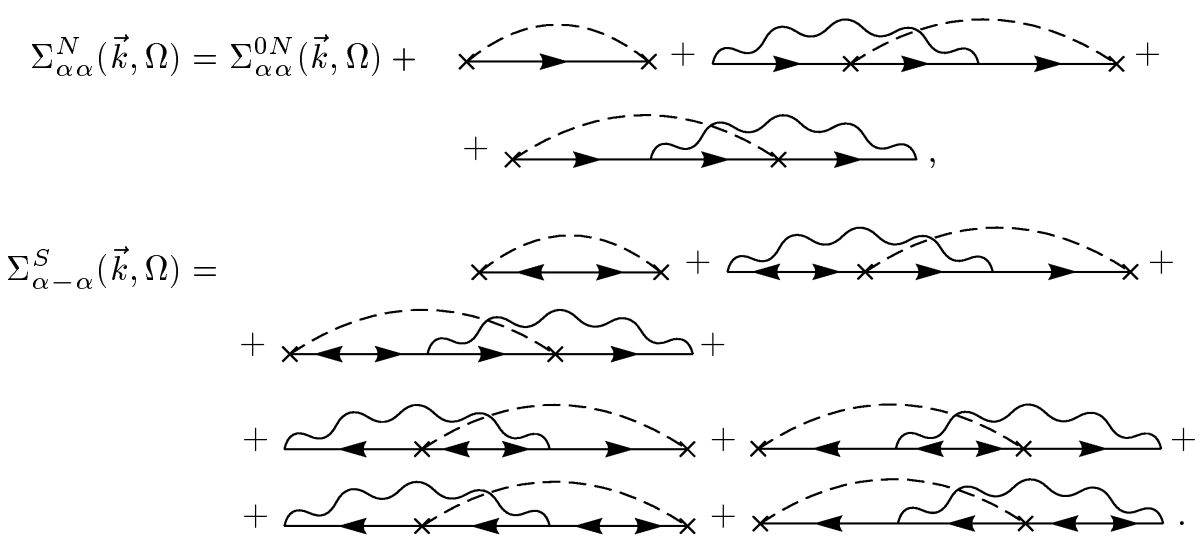

где $\Sigma_{N}^{0}$ и $\Sigma_{S}^{0}$ определяются диаграммами (1) и (2), соответственно. Прямые линии со стрелками соответствуют полным функциям Грина, нормальным $(\rightarrow)$ и аномальным $(\leftrightarrow)$, усредненным по положениям атомов примеси и ориентациям их спинов, волнистые - фононной функции Грина, а штриховые - электрон-примесному взаимодействию.

Отметим, что диаграммы (9) и (10) определяют вклад в массовые операторы при $T \sim T_{\text {c }}$. Если рассматривать всю температурную область $0<T<T_{\text {c }}$, то указанные выше выражения, вообе говоря, должны быть дополнены соответствующими диаграммами, содержащими по две аномальные функции Грина. В приближении слабой связи $\left(\Delta / \omega_{0} \ll 1\right)$ вклад этих диаграмм пренебрежимо мал, что позволяет считать выражения (9) и (10) справедливыми во всем температурном интервале, где сушествует сверхпроводимость.

Выберем для простоты эйнштейновский спектр и представим фононную функцию Грина в виде

$$
D\left(\vec{p}-\vec{p}_{1}, \Omega-\Omega_{1}\right)=-\left|g_{\vec{p} \vec{p}_{1}}\right|^{2} \frac{\omega_{0}}{\left(\Omega-\Omega_{1}\right)^{2}+\omega_{0}^{2}}=\left|g_{\vec{p} \vec{p}_{1}}\right|^{2} D\left(\Omega, \Omega_{1}\right)
$$

где $g_{\vec{p} \vec{p}_{1}}$ - константа электрон-фононного взаимодействия, $\omega_{0}$ - максимальная фононная частота.

Исследование влияния сильных электронных коррелящий на электрон-фононное или электрон-примесное взаимодействие [31], [32] показывает, что с ростом обменного импульса $q$ эти взаимодействия слабо растут, а затем резко убывают. Эти результаты позволяют приблизительно представить вышеуказанные величины в виде

$$
\begin{gathered}
\left|g_{\vec{p} \vec{p}_{1}}\right|^{2}=\left(\frac{2 k_{\mathrm{F}}}{q_{c}}\right)^{2} g^{2} \theta\left(q_{c}-\left|\vec{p}-\vec{p}_{1}\right|\right), \\
V_{i}\left(\vec{p}-\vec{p}_{1}\right) V_{j}\left(\vec{p}_{1}-\vec{p}\right)=V_{i} V_{j}\left(\frac{2 k_{\mathrm{F}}}{q_{c 1}}\right)^{2} \theta\left(q_{c 1}-\left|\vec{p}-\vec{p}_{1}\right|\right), \quad i, j=1,2,
\end{gathered}
$$


где $q_{c}$ и $q_{c 1}$ - импульсы обрезания электрон-фононного и электрон-примесного взаимодействий, соответственно. Множитель $\left(2 k_{\mathrm{F}} / q_{c}\right)^{2}$ вводится, чтобы в результате усреднения выражения (12) по поверхности Ферми получить $g^{2}$ и, следовательно, в этой модели константа $\lambda=N_{0} g^{2}$ не зависит от $q_{c}$ в согласии с результатами работы [31]. Такая же ситуация имеет место для примесного потенциала (13).

Используя соотношения (12) и (13), введем определения вершинных и пересекаюшихся функций:

$$
\begin{aligned}
& P_{V}^{\alpha \alpha}\left(\vec{p} \vec{p}_{1}, \Omega \Omega_{1}\right)=-\frac{1}{\beta V N_{0}}\left(\frac{2 k_{\mathrm{F}}}{q_{c}}\right)^{2} \sum_{\vec{p}_{2}, \Omega_{2}} \theta\left(q_{c}-\left|\vec{p}-\vec{p}_{2}\right|\right) D\left(\Omega, \Omega_{2}\right) \times \\
& \times \bar{G}_{\alpha \alpha}\left(\vec{p}_{2}, \Omega_{2}\right) \bar{G}_{\alpha \alpha}\left(\vec{p}_{1}+\vec{p}_{2}-\vec{p}, \Omega_{1}+\Omega_{2}-\Omega\right), \\
& P_{c}^{\alpha-\alpha}\left(\vec{p} \vec{p}_{1}, \Omega \Omega_{1}\right) \approx-\frac{1}{\beta V N_{0}}\left(\frac{2 k_{\mathrm{F}}}{q_{c}}\right)^{2} \sum_{\vec{p}_{2}, \Omega_{2}} \theta\left(q_{c}-\left|\vec{p}-\vec{p}_{2}\right|\right) D\left(\Omega, \Omega_{2}\right) \times \\
& \times \bar{G}_{\alpha \alpha}\left(\vec{p}_{2}, \Omega_{2}\right) \bar{G}_{-\alpha-\alpha}\left(\vec{p}_{2}-\vec{p}_{1}-\vec{p}, \Omega_{2}-\Omega_{1}-\Omega\right), \\
& R_{V}^{\alpha \alpha}\left(\vec{p} \vec{p}_{1}, \Omega \Omega_{1}\right) \approx \frac{1}{N_{0} V}\left(\frac{2 k_{\mathrm{F}}}{q_{c 1}}\right)^{2} \sum_{\vec{p}_{2}} W_{+}^{\alpha} \theta\left(q_{c 1}-\left|\vec{p}-\vec{p}_{2}\right|\right) \times \\
& \times \bar{G}_{\alpha \alpha}\left(\vec{p}_{2}, \Omega\right) \bar{G}_{\alpha \alpha}\left(\vec{p}_{1}+\vec{p}_{2}-\vec{p}, \Omega_{1}\right), \\
& R_{c}^{\alpha-\alpha}\left(\vec{p} \vec{p}_{1}, \Omega \Omega_{1}\right) \approx \frac{1}{N_{0} V}\left(\frac{2 k_{\mathrm{F}}}{q_{c 1}}\right)^{2} \sum_{\vec{p}_{2}} W_{-} \theta\left(q_{c 1}-\left|\vec{p}-\vec{p}_{2}\right|\right) \times \\
& \times \bar{G}_{\alpha \alpha}\left(\vec{p}_{2}, \Omega_{1}\right) \bar{G}_{-\alpha-\alpha}\left(\vec{p}_{2}-\vec{p}_{1}-\vec{p},-\Omega\right), \\
& K_{V}^{-\alpha-\alpha}\left(\vec{p} \vec{p}_{1}, \Omega \Omega_{1}\right)=\frac{1}{N_{0} V}\left(\frac{2 k_{\mathrm{F}}}{q_{c 1}}\right)^{2} \sum_{\vec{p}_{2}} U \theta\left(q_{c 1}-\left|\vec{p}-\vec{p}_{2}\right|\right) \times \\
& \times \bar{G}_{-\alpha-\alpha}\left(\vec{p}_{2}, \Omega\right) \bar{G}_{-\alpha-\alpha}\left(\vec{p}_{1}+\vec{p}_{2}-\vec{p}, \Omega_{1}\right), \\
& K_{c}^{\alpha \alpha}\left(\vec{p} \vec{p}_{1}, \Omega \Omega_{1}\right) \approx \frac{1}{N_{0} V}\left(\frac{2 k_{\mathrm{F}}}{q_{c 1}}\right)^{2} \sum_{\vec{p}_{2}} U \theta\left(q_{c 1}-\left|\vec{p}-\vec{p}_{2}\right|\right) \times \\
& \times \bar{G}_{\alpha \alpha}\left(\vec{p}_{2}, \Omega_{1}\right) \bar{G}_{\alpha \alpha}\left(\vec{p}_{2}-\vec{p}_{1}-\vec{p},-\Omega\right),
\end{aligned}
$$

где

$$
\begin{gathered}
W_{+}^{\alpha}=\Gamma_{1}+2 \Gamma_{12} \sigma_{\alpha \alpha}^{z}+\Gamma_{\mathrm{ex}}\left\langle S_{z}^{2}\right\rangle, \\
W_{-}=\Gamma_{1}-\Gamma_{\mathrm{ex}}\left\langle S_{z}^{2}\right\rangle, \quad U=\Gamma_{\mathrm{ex}}\left\langle S_{x}^{2}\right\rangle+\Gamma_{\mathrm{ex}}\left\langle S_{y}^{2}\right\rangle, \quad \Gamma_{1}=N_{0} c V_{1}^{2} \pi, \\
\Gamma_{\mathrm{ex}}=\pi c N_{0} V_{2}^{2}, \quad \Gamma_{12}=\pi V_{1} V_{2}\left\langle S_{z}\right\rangle c N_{0} .
\end{gathered}
$$

Величины $P_{V}$ и $P_{c}$ появляются вследствие учета соответственно вершинных и пересекаюшихся диаграмм по электрон-фононному взаимодействию, все остальные связаны с пересечением линий электрон-фононных с электрон-примесным взаимодействием. При этом в пересекаюшихся диаграммах, где смешивается суммирование по $\vec{p}_{1}$ и $\vec{p}_{2}$, ограничиваемся приближением [30] $D\left(\vec{p}_{1}-\vec{p}_{2}, \Omega_{1}-\Omega_{2}\right) \rightarrow D\left(\vec{p}-\vec{p}_{2}, \Omega-\Omega_{2}\right), W_{ \pm}\left(\vec{p}_{1}-\vec{p}_{2}\right) \rightarrow$ 
$W_{ \pm}\left(\vec{p}-\vec{p}_{2}\right)$. На основании формул $(9),(10)$ и $(1),(2)$, а также определений $(14)-(16)$ имеем

$$
\begin{aligned}
\Sigma_{\alpha \alpha}^{N}(\vec{p}, \Omega)= & \frac{1}{\beta V} \sum_{\vec{p}_{1}, \Omega_{1}} \bar{V}_{N}^{\alpha}\left(\vec{p}, \vec{p}_{1}, \Omega, \Omega_{1}\right) \bar{G}_{\alpha \alpha}\left(\vec{p}_{1}, \Omega_{1}\right)+\frac{1}{V} \sum_{\vec{p}_{1}} \bar{W}_{+}^{\alpha}\left(\vec{p}, \vec{p}_{1}, \Omega\right) \bar{G}_{\alpha \alpha}\left(\vec{p}_{1}, \Omega\right)+ \\
& +\frac{1}{V} \sum_{\vec{p}_{1}} \bar{U}^{\alpha}\left(\vec{p}, \vec{p}_{1}, \Omega\right) \bar{G}_{-\alpha-\alpha}\left(\vec{p}_{1}, \Omega\right), \\
\Sigma_{\alpha-\alpha}^{S}(\vec{p}, \Omega)= & \frac{1}{\beta V} \sum_{\vec{p}_{1}, \Omega_{1}} \bar{V}_{S}^{\alpha}\left(\vec{p}, \vec{p}_{1}, \Omega, \Omega_{1}\right) \bar{F}_{\alpha-\alpha}\left(\vec{p}_{1}, \Omega_{1}\right)+ \\
& +\frac{1}{\beta V} \sum_{\vec{p}_{1}, \Omega_{1}} \bar{V}_{S 1}^{\alpha}\left(\vec{p}, \vec{p}_{1}, \Omega, \Omega_{1}\right) \bar{F}_{-\alpha \alpha}\left(\vec{p}_{1}, \Omega_{1}\right)+ \\
& +\frac{1}{V} \sum_{\vec{p}_{1}} \bar{W}_{-}^{\alpha}\left(\vec{p}, \vec{p}_{1}, \Omega\right) \bar{F}_{\alpha-\alpha}\left(\vec{p}_{1}, \Omega\right)+ \\
& +\frac{1}{V} \sum_{\vec{p}_{1}} \bar{U}^{\alpha}\left(\vec{p}, \vec{p}_{1}, \Omega\right) \bar{F}-\alpha \alpha\left(\vec{p}_{1}, \Omega\right),
\end{aligned}
$$

где

$$
\begin{aligned}
& \bar{V}_{N}^{\alpha}\left(\vec{p}, \vec{p}_{1}, \Omega, \Omega_{1}\right)=-g^{2} D\left(\Omega, \Omega_{1}\right) \theta\left(q_{c}-\left|\vec{p}-\vec{p}_{1}\right|\right) \times \\
& \times\left(\frac{2 k_{\mathrm{F}}}{q_{c}}\right)^{2}\left[1+\lambda P_{V}^{\alpha \alpha}\left(\vec{p}, \vec{p}_{1}, \Omega, \Omega_{1}\right)\right] \\
& \bar{V}_{S}^{\alpha}\left(\vec{p}, \vec{p}_{1}, \Omega, \Omega_{1}\right)=-g^{2} D\left(\Omega, \Omega_{1}\right)\left(\frac{2 k_{\mathrm{F}}}{q_{c}}\right)^{2} \theta\left(q_{c}-\left|\vec{p}-\vec{p}_{1}\right|\right) \times \\
& \times\left[1+\lambda P_{V}^{\alpha \alpha}\left(\vec{p}, \vec{p}_{1}, \Omega, \Omega_{1}\right)+\lambda P_{V}^{-\alpha-\alpha}\left(-\vec{p},-\vec{p}_{1},-\Omega,-\Omega_{1}\right)+\right. \\
& \left.+\lambda P_{c}^{\alpha-\alpha}\left(\vec{p}, \vec{p}_{1}, \Omega, \Omega_{1}\right)\right]- \\
& -\lambda D\left(\Omega, \Omega_{1}\right)\left(\frac{2 k_{\mathrm{F}}}{q_{c}}\right)^{2} \theta\left(q_{c}-\left|\vec{p}-\vec{p}_{1}\right|\right)\left[R_{V}^{\alpha \alpha}\left(\vec{p}, \vec{p}_{1}, \Omega, \Omega_{1}\right)+\right. \\
& +R_{V}^{-\alpha-\alpha}\left(-\vec{p},-\vec{p}_{1},-\Omega,-\Omega_{1}\right)+ \\
& +R_{c}^{\alpha-\alpha}\left(\vec{p}, \vec{p}_{1}, \Omega, \Omega_{1}\right)+R_{c}^{\alpha-\alpha}\left(\vec{p}, \vec{p}_{1}, \Omega, \Omega_{1}\right)+ \\
& \left.+K_{V}^{-\alpha-\alpha}\left(\vec{p}, \vec{p}_{1}, \Omega, \Omega_{1}\right)+K_{V}^{\alpha \alpha}\left(-\vec{p},-\vec{p}_{1},-\Omega,-\Omega_{1}\right)\right], \\
& \bar{V}_{S 1}^{\alpha}\left(\vec{p}, \vec{p}_{1}, \Omega, \Omega_{1}\right)=-g^{2}\left(\frac{2 k_{\mathrm{F}}}{q_{c}}\right)^{2} \theta\left(q_{c}-\left|\vec{p}-\vec{p}_{1}\right|\right) D\left(\Omega, \Omega_{1}\right) \times \\
& \times\left[K_{c}^{\alpha \alpha}\left(\vec{p}, \vec{p}_{1}, \Omega, \Omega_{1}\right)+K_{c}^{-\alpha-\alpha}\left(\vec{p}, \vec{p}_{1}, \Omega, \Omega_{1}\right)\right], \\
& \bar{W}_{ \pm}^{\alpha}\left(\vec{p}, \vec{p}_{1}, \Omega\right)=W_{ \pm}\left[1+\lambda P_{V}^{\alpha \alpha}\left(\vec{p}, \vec{p}_{1}, \Omega, \Omega\right)+\lambda P_{V}^{-\alpha-\alpha}\left(-\vec{p},-\vec{p}_{1},-\Omega,-\Omega\right)\right] \times \\
& \times\left(\frac{2 k_{\mathrm{F}}}{q_{c 1}}\right)^{2} \theta\left(q_{c 1}-\left|\vec{p}-\vec{p}_{1}\right|\right) \\
& \bar{U}^{\alpha}\left(\vec{p}, \vec{p}_{1}, \Omega\right)=U\left[1+\lambda P_{V}^{\alpha-\alpha}\left(\vec{p}, \vec{p}_{1}, \Omega, \Omega\right)+\lambda P_{V}^{-\alpha \alpha}\left(\vec{p}, \vec{p}_{1}, \Omega, \Omega\right)\right] \times \\
& \times\left(\frac{2 k_{\mathrm{F}}}{q_{c 1}}\right)^{2} \theta\left(q_{c 1}-\left|\vec{p}-\vec{p}_{1}\right|\right) \text {. }
\end{aligned}
$$


Выражения (18) и (19) существенно усложняются по сравнению со случаем адиабатических систем за счет перенормировки электрон-фононного и электрон-примесного взаимодействий. Наряду с этим из-за спинового упорядочения примеси $\left(\left\langle S_{z}\right\rangle \neq 0\right)$ происходит "расщепление" примесных вкладов (в (18) возникают два примесных члена, а в $(19)$ - три), которые в парамагнитном пределе $\left(\left\langle S_{z}\right\rangle=0\right)$ из-за равенств $\bar{G}_{\alpha \alpha}=$ $\bar{G}_{-\alpha-\alpha}$ и $\bar{F}_{\alpha-\alpha}=-\bar{F}_{-\alpha \alpha}$ складываются, приводя к известному результату [34].

На основании формул (18) и (19) можно получить известные результаты для чистой неадиабатической системы [30], полагая $c=0$, а также для неадиабатической системы с неупорядоченным магнитным спином (парамагнитная фаза $\left\langle S_{z}\right\rangle=0$ ) [34]. Полагая же все вершинные поправки равными нулю, приходим к адиабатической теории сверхпроводимости в ферромагнитных сплавах [17], [35].

После усреднения по поверхности Ферми выражений (18) и (19) (для случая $\alpha=\uparrow$ ) получаем

$$
\begin{aligned}
\Sigma_{\uparrow \downarrow}^{N}(\Omega)= & \frac{1}{\beta V} \sum_{\vec{p}_{1}, \Omega_{1}} V_{N}^{\uparrow}\left(\Omega, \Omega_{1}\right) \bar{G}_{\uparrow \uparrow}\left(\vec{p}_{1}, \Omega_{1}\right)+ \\
& +\frac{1}{V} \sum_{\vec{p}_{1}} \bar{W}_{+}^{\uparrow}(\Omega) \bar{G}_{\uparrow \uparrow}\left(\vec{p}_{1}, \Omega\right)+\frac{1}{V} \sum_{\vec{p}_{1}} \bar{U}^{\uparrow}(\Omega) \bar{G}_{\downarrow \downarrow}\left(\vec{p}_{1}, \Omega\right), \\
\Sigma_{\uparrow \downarrow}^{S}(\Omega)= & \frac{1}{\beta V} \sum_{\vec{p}_{1}, \Omega_{1}} \bar{V}_{S}^{\uparrow}\left(\Omega, \Omega_{1}\right) F_{\uparrow \downarrow}\left(\vec{p}_{1}, \Omega_{1}\right)+\frac{1}{\beta V} \sum_{\vec{p}_{1}} \bar{V}_{S 1}^{\uparrow}\left(\Omega, \Omega_{1}\right) F_{\downarrow \uparrow}\left(\vec{p}_{1}, \Omega_{1}\right)+ \\
& +\frac{1}{V} \sum_{\vec{p}_{1}} \bar{W}_{-}^{\uparrow}(\Omega) F_{\uparrow \downarrow}\left(\vec{p}_{1}, \Omega\right)+\frac{1}{V} \sum_{\vec{p}_{1}} \bar{U}^{\uparrow}(\Omega) F_{\downarrow \uparrow}\left(\vec{p}_{1}, \Omega\right) .
\end{aligned}
$$

Коэффициенты при функциях Грина в этих выражениях представляют собой выражения (20)-(24), усредненные по поверхности Ферми.

Введем определения

$$
\Sigma_{\uparrow \uparrow}^{N}(\Omega)=\Sigma_{+}^{N^{(1)}}(\Omega)+\Sigma_{+}^{N^{(2)}}(\Omega), \quad \Sigma_{\downarrow \downarrow}^{N}(\Omega)=\Sigma_{-}^{N^{(1)}}(\Omega)+\Sigma_{-}^{N^{(2)}}(\Omega),
$$

где

$$
\Sigma_{+}^{N^{(1)}}(\Omega)=\Sigma_{-}^{N^{(1)}}(-\Omega), \quad \Sigma_{+}^{N^{(2)}}(\Omega)=-\Sigma_{-}^{N^{(2)}}(-\Omega)
$$

а также

$$
\Sigma_{\uparrow \downarrow}^{S}(\Omega)=\widetilde{\Sigma}_{1+}(\Omega)+i \widetilde{\Sigma}_{2+}(\Omega), \quad \Sigma_{\downarrow \uparrow}^{S}(\Omega)=\widetilde{\Sigma}_{1-}(\Omega)+i \widetilde{\Sigma}_{2-}(\Omega) .
$$

С использованием этих определений решения системы уравнений для функций Грина (8) приводятся к виду

$$
\begin{array}{ll}
\bar{G}_{\uparrow \uparrow}(\vec{p}, \Omega)=-\frac{i \widetilde{\Omega}_{+}+\tilde{\varepsilon}_{p+}}{D_{+}}, & \bar{F}_{\uparrow \downarrow}(\vec{p}, \Omega)=\frac{\widetilde{\Sigma}_{1+}+i \widetilde{\Sigma}_{2+}}{D_{+}}, \\
\bar{G}_{\downarrow \downarrow}(\vec{p}, \Omega)=-\frac{i \widetilde{\Omega}_{-}+\tilde{\varepsilon}_{p-}}{D_{-}}, & \bar{F}_{\downarrow \uparrow}(\vec{p}, \Omega)=-\frac{\widetilde{\Sigma}_{1-}+i \widetilde{\Sigma}_{2-}}{D_{-}},
\end{array}
$$


где

$$
\begin{gathered}
D_{ \pm}=\widetilde{\Omega}_{ \pm}^{2}+\tilde{\varepsilon}_{p \pm}^{2}+\widetilde{\Sigma}_{1 \pm}^{2}+\widetilde{\Sigma}_{2 \pm}^{2}, \\
\widetilde{\Omega}_{ \pm}=\Omega \pm i I+i \Sigma_{N \pm}^{(2)}(\Omega)=Z_{ \pm} \Omega \pm i I+\left(\bar{\Gamma}_{1}+\bar{\Gamma}_{\mathrm{ex}}\left\langle S_{z}^{2}\right\rangle\right) \frac{\widetilde{\Omega}_{ \pm} S_{ \pm}}{\sqrt{D_{ \pm}}} \varphi_{ \pm}+ \\
+\bar{\Gamma}_{\mathrm{ex}}\left(\left\langle S_{x}^{2}\right\rangle+\left\langle S_{y}^{2}\right\rangle\right) \frac{\widetilde{\Omega}_{\mp} S_{\mp}}{\sqrt{D_{\mp}}} \varphi_{\mp}, \\
\tilde{\varepsilon}_{p \pm}=\varepsilon_{p}+\Sigma_{N \pm}^{(1)}(\Omega)=\varepsilon_{p} \mp i\left\langle S_{z}\right\rangle \bar{\Gamma}_{12} \frac{\widetilde{\Omega}_{ \pm} S_{ \pm}}{\sqrt{D_{ \pm}}} \varphi_{ \pm}, \\
\widetilde{\Sigma}_{1 \pm}=\Sigma_{2}+\left(\bar{\Gamma}_{1}-\bar{\Gamma}_{\mathrm{ex}}\left\langle S_{z}^{2}\right\rangle\right) \frac{\widetilde{\Sigma}_{1 \pm} S_{ \pm}}{\sqrt{D_{ \pm}}} \varphi_{ \pm}-\bar{\Gamma}_{\mathrm{ex}}\left(\left\langle S_{x}^{2}\right\rangle+\left\langle S_{y}^{2}\right\rangle\right) \frac{\widetilde{\Sigma}_{1 \mp} S_{\mp}}{\sqrt{D_{\mp}}} \varphi_{\mp}, \\
\widetilde{\Sigma}_{2 \pm}=\Sigma_{2}+\left(\bar{\Gamma}_{1}-\bar{\Gamma}_{\mathrm{ex}}\left\langle S_{z}^{2}\right\rangle\right) \frac{\widetilde{\Sigma}_{2 \pm} S_{ \pm}}{\sqrt{D_{ \pm}}} \varphi_{ \pm}-\bar{\Gamma}_{\mathrm{ex}}\left(\left\langle S_{x}^{2}\right\rangle+\left\langle S_{y}^{2}\right\rangle\right) \frac{\widetilde{\Sigma}_{2 \mp} S_{\mp}}{\sqrt{D_{\mp}}} \varphi_{\mp},
\end{gathered}
$$

здесь

$$
\begin{gathered}
Z_{ \pm}=1+\frac{1}{\Omega} \frac{\pi}{\beta} \sum_{\Omega_{1}} V_{N}\left(\Omega, \Omega_{1}\right) \frac{\widetilde{\Omega}_{1+} S_{ \pm}}{\sqrt{D_{ \pm}}} \varphi_{ \pm}, \\
\bar{\Gamma}_{i}=\Gamma_{i}\left[1+\lambda P_{V}^{++}\left(Q_{c}, \Omega, \Omega\right)+\lambda P_{V}^{--}\left(Q_{c},-\Omega,-\Omega\right)\right], \\
\bar{\Gamma}_{12}=\Gamma_{12}\left[1+\lambda P_{V}^{+-}\left(Q_{c}, \Omega, \Omega\right)+\lambda P_{V}^{-+}\left(Q_{c},-\Omega,-\Omega\right)\right], \\
S_{ \pm}=\operatorname{sgn}\left(\operatorname{Re} \sqrt{D_{ \pm}}\right), \quad \varphi_{ \pm}=\frac{2}{\pi} \operatorname{arctg} \frac{E S_{ \pm}}{2 \sqrt{D_{ \pm}}} .
\end{gathered}
$$

\section{3. ВЕРШИННЫЕ ФУНКЦИИ И ТЕМПЕРАТУРА СВЕРХПРОВОДЯЩЕГО ПЕРЕХОДА}

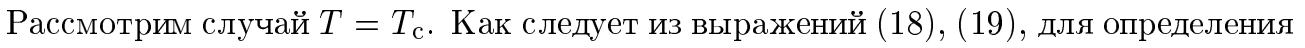
величины $T_{\mathrm{c}}$ следует выполнить линеаризацию по массовому оператору $\Sigma_{S}$ и вычислить все вершинные и пересекаюшиеся функции, которые содержат сложную зависимость от обменного импульса и частоты, как и в случае чистого вешества [30], а также дополнительные зависимости от внутреннего магнитного поля и концентрации примеси. Вычисление этих функций в общем виде из-за наличия угловых зависимостей является довольно непростой задачей. С целью получить аналитические выражения рассматриваем приближение слабой связи $\left(T_{\mathrm{c}} / \omega_{0} \ll 1, I / E \ll 1, I / \omega_{0} \ll 1\right)$, а также условия $\Gamma_{1} \ll \omega_{0}, \Gamma_{\text {ex }} \ll \omega_{0}$, которые выполняются при небольших концентрашиях примеси, при которых сушествует сверхпроводящее состояние. Используем метод прямого вычисления [9], [30] (см. приложение). При этом получаем

$$
\begin{aligned}
P_{V, c}^{\uparrow \uparrow}\left(Q_{c}, \Omega, \Omega_{1}\right) & =\left(\frac{2 k_{\mathrm{F}}}{q_{c}}\right)^{2}\left\langle P_{V, c}^{\uparrow \uparrow}\left(\vec{p}, \vec{p}_{1}, \Omega, \Omega_{1}\right) \theta\left(q_{c}-\left|\vec{p}-\vec{p}_{1}\right|\right)\right\rangle_{\mathrm{FS}}= \\
& =P_{V, c}^{0}\left(Q_{c}, \Omega, \Omega_{1}\right)+O\left(\frac{I}{\omega_{0}}\right)+O\left(\frac{\Gamma_{1} \pm \Gamma}{\omega_{0}}\right),
\end{aligned}
$$

6 Теоретическая и математическая физика, т. 135, № 1, 2003 г. 


$$
\begin{aligned}
R_{V, c}^{\uparrow \uparrow}\left(Q_{c}, \Omega, \Omega_{1}\right) & =\left(\frac{2 k_{\mathrm{F}}}{q_{c 1}}\right)^{2}\left\langle R_{V, c}^{\uparrow \uparrow}\left(\vec{p}, \vec{p}_{1}, \Omega, \Omega_{1}\right) \theta\left(q_{c 1}-\left|\vec{p}-\vec{p}_{1}\right|\right)\right\rangle_{\mathrm{FS}} \approx \\
& \approx O\left(\frac{I}{\omega_{0}}\right)+O\left(\frac{\Gamma_{1} \pm \Gamma}{\omega_{0}}\right), \\
K_{V, c}^{\uparrow \uparrow}\left(Q_{c}, \Omega, \Omega_{1}\right) & \approx O\left(\frac{I}{\omega_{0}}\right)+O\left(\frac{\Gamma_{1} \pm \Gamma}{\omega_{0}}\right),
\end{aligned}
$$

$P_{V, c}^{0}\left(Q_{c}, \Omega, \Omega_{1}\right)$ - соответственно вершинная и пересекаюшаяся функции для беспримесной электрон-фононной системы [30] (см. также приложение), $\Gamma=\Gamma_{\text {ех }} S(S+1)$, а индекс FS означает усреднение по поверхности Ферми. На основании этих результатов можно записать

$$
\begin{gathered}
\bar{V}_{N}\left(\Omega, \Omega_{1}\right) \approx-g^{2} D\left(\Omega, \Omega_{1}\right)\left[1+\lambda P_{V}^{0}\left(Q_{c}, \Omega, \Omega_{1}\right)\right] \\
\bar{V}_{S}\left(\Omega, \Omega_{1}\right)=-g^{2} D\left(\Omega, \Omega_{1}\right)\left[1+2 \lambda P_{V}^{0}\left(Q_{c}, \Omega, \Omega_{1}\right)+\lambda P_{c}^{0}\left(Q_{c}, \Omega, \Omega_{1}\right)\right] \\
\bar{W}_{ \pm}^{\uparrow}(\Omega)=W_{ \pm}\left[1+2 \lambda P_{V}^{0}\left(Q_{c}, \Omega, \Omega\right)\right] \\
\bar{U}^{\uparrow}(\Omega)=U\left[1+2 \lambda P_{V}^{0}\left(Q_{c}, \Omega, \Omega\right)\right] .
\end{gathered}
$$

Выполнив в уравнениях (18) и (19) после линеаризации интегрирование по энергии обычным способом $\left((1 / V) \sum_{p_{1}} \ldots \rightarrow N_{0} \int_{-E / 2}^{E / 2} \cdots d \varepsilon_{\vec{p}_{1}}\right.$, где $N_{0}$ - плотность электронных состояний на поверхности Ферми), получаем самосогласованную систему уравнений для определения величины $T_{\mathrm{c}}$ :

$$
\begin{aligned}
& \Sigma_{1}(\Omega)+i \Sigma_{2}(\Omega)= \frac{2 \lambda}{\beta} \sum_{\Omega_{1}} \frac{\omega_{0}^{2}}{\left(\Omega-\Omega_{1}\right)^{2}+\omega_{0}^{2}}\left[1+2 \lambda P_{V}^{0}\left(Q_{c}, \Omega, \Omega_{1}\right)+\right. \\
&\left.+\lambda P_{c}^{0}\left(Q_{c}, \Omega, \Omega_{1}\right)\right] \frac{\widetilde{\Sigma}_{1+}\left(\Omega_{1}\right)+i \widetilde{\Sigma}_{2+}\left(\Omega_{1}\right)}{\widetilde{\Omega}_{1+}} \operatorname{arctg} \frac{E}{2 \Omega_{1} Z}, \\
& \widetilde{\Omega}_{ \pm}=Z \Omega \pm i I+\left(\widetilde{\Gamma}_{1}+\widetilde{\Gamma}_{\mathrm{ex}} S(S+1)\right), \\
& \widetilde{\Sigma}_{1 \pm}=\Sigma_{1}+\left(\widetilde{\Gamma}_{1}-\widetilde{\Gamma}_{\mathrm{ex}}\left\langle S_{z}^{2}\right\rangle\right) \frac{\Sigma_{1 \pm}}{\widetilde{\Omega}_{ \pm}}-\widetilde{\Gamma}_{\mathrm{ex}}\left(\left\langle S_{z}^{2}\right\rangle+\left\langle S_{y}^{2}\right\rangle\right) \frac{\widetilde{\Sigma}_{1 \mp}}{\widetilde{\Omega}_{\mp}}
\end{aligned}
$$

где

$$
\begin{gathered}
Z=1+\frac{2 \lambda}{\beta} \sum_{\Omega_{1}} \frac{\omega_{0}^{2}}{\left(\Omega-\Omega_{1}\right)^{2}+\omega_{0}^{2}}\left[1+\lambda P_{V}^{0}\left(Q_{c}, \Omega, \Omega_{1}\right)\right] \operatorname{arctg} \frac{E}{2 \Omega}, \\
\widetilde{\Gamma}_{j}=\bar{\Gamma}_{j} \frac{2}{\pi} \operatorname{arctg} \frac{E}{2 \Omega}, \quad \bar{\Gamma}_{j}=\Gamma_{j}\left[1+2 \lambda P_{V}^{0}\left(Q_{c}, \Omega, \Omega\right)\right], \quad j=1, \text { ex. }
\end{gathered}
$$

Заменив в последней формуле $(34)$ индекс $1 \rightarrow 2$, получим систему уравнений для $\widetilde{\Sigma}_{2 \pm}$. Решение этой системы и системы уравнений (34) приводит к следующему результату:

$$
\widetilde{\Sigma}_{1+}+i \widetilde{\Sigma}_{2+}=F(\Omega)\left(\Sigma_{1}+i \Sigma_{2}\right)
$$


где

$$
F(\Omega)=\frac{\widetilde{\Omega}_{+}\left[\widetilde{\Omega}_{-}-\left(\widetilde{\Gamma}_{1}+\widetilde{\Gamma}_{\mathrm{ex}}\left(\left\langle S_{x}^{2}\right\rangle+\left\langle S_{y}^{2}\right\rangle-\left\langle S_{z}^{2}\right\rangle\right)\right)\right]}{\widetilde{\Omega}_{+} \widetilde{\Omega}_{-}-\left(\widetilde{\Gamma}_{1}-\widetilde{\Gamma}_{\mathrm{ex}}\left\langle S_{z}^{2}\right\rangle\right)\left(\widetilde{\Omega}_{+}+\widetilde{\Omega}_{-}\right)+\left(\widetilde{\Gamma}_{1}+\widetilde{\Gamma}_{\mathrm{ex}}\left\langle S_{z}^{2}\right\rangle\right)^{2}-\left(\widetilde{\Gamma}_{\mathrm{ex}}\left(\left\langle S_{x}^{2}\right\rangle+\left\langle S_{y}^{2}\right\rangle\right)\right)^{2}} .
$$

С использованием (36) уравнение для параметра порядка приобретает вид

$$
\begin{aligned}
\Sigma_{1}(\Omega)+i \Sigma_{2}(\Omega)= & \frac{2}{\beta} \lambda \sum_{\Omega_{1}} \frac{\omega_{0}^{2}}{\left(\Omega-\Omega_{1}\right)^{2}+\omega_{0}^{2}}\left[1+2 \lambda P_{V}^{0}\left(Q_{c}, \Omega, \Omega_{1}\right)+\lambda P_{c}^{0}\left(Q_{c}, \Omega, \Omega_{1}\right)\right] \times \\
& \times \frac{F\left(\Omega_{1}\right)}{\widetilde{\Omega}_{1+}}\left[\Sigma_{1}\left(\Omega_{1}\right)+i \Sigma_{2}\left(\Omega_{1}\right)\right] \operatorname{arctg} \frac{E}{2 \Omega_{1} Z} .
\end{aligned}
$$

В соответствии с приближением, принятым в [30], заменим величины $P_{V}^{0}$ и $P_{c}^{0}$ их значениями при $\Omega=0$ и $\Omega_{1}=\omega_{0}$, а также введем обозначения

$$
\begin{aligned}
\lambda_{\triangle} & =\lambda\left[1+2 \lambda P_{V}^{0}\left(Q_{c}, 0, \omega_{0}\right)+\lambda P_{c}^{0}\left(Q_{c}, 0, \omega_{0}\right)\right], \\
\lambda_{z} & =\lambda\left[1+\lambda P_{V}^{0}\left(Q_{c}, 0, \omega_{0}\right)\right] .
\end{aligned}
$$

В уравнении (38) вьполним преобразования, которые используются в теории сверхпроводимости с электрон-фононным взаимодействием [11], [34]. Выполним замену

$$
\frac{\omega_{0}^{2}}{\left(\Omega-\Omega_{1}\right)^{2}+\omega_{0}^{2}} \rightarrow \frac{\omega_{0}^{2}}{\Omega^{2}+\omega_{0}^{2}} \frac{\omega_{0}^{2}}{\Omega_{1}^{2}+\omega_{0}^{2}}
$$

Введем также обозначение

$$
A=\frac{2 \lambda}{\beta} \sum_{\Omega_{1}} \frac{\omega_{0}^{2}}{\Omega_{1}^{2}+\omega_{0}^{2}} \frac{F\left(\Omega_{1}\right)}{\widetilde{\Omega}_{1+}}\left[\Sigma_{1}\left(\Omega_{1}\right)+i \Sigma_{2}\left(\Omega_{1}\right)\right] \operatorname{arctg} \frac{E}{2 Z \Omega_{1}} .
$$

В результате имеем

$$
\Sigma_{1}(\Omega)+i \Sigma_{2}(\Omega)=\frac{\omega_{0}^{2}}{\Omega^{2}+\omega_{0}^{2}} A .
$$

Подставляя (41) в (40), получаем уравнение для определения температуры сверхпроводяшего перехода

$$
1=\frac{2}{\beta_{c}} \lambda_{\triangle} \sum_{\Omega_{1}} \frac{\omega_{0}^{4}}{\left(\Omega_{1}^{2}+\omega_{0}^{2}\right)^{2}} \frac{F\left(\Omega_{1}\right)}{\widetilde{\Omega}_{1+}} \operatorname{arctg} \frac{E}{2 Z \Omega_{1}} .
$$

Это уравнение в приближении слабой связи $\left(T_{\mathrm{c}} \ll \omega_{0}\right)$ можно привести к виду

$$
\ln \frac{T_{\mathrm{c}}}{T_{\mathrm{c} 0}}=\frac{2}{\beta_{c}} Z \sum_{\Omega_{1}} \frac{\omega_{0}^{4}}{\left(\Omega_{1}^{2}+\omega_{0}^{2}\right)^{2}}\left[\frac{F\left(\Omega_{1}\right)}{\widetilde{\Omega}_{1+}}-\frac{1}{Z \Omega_{1}}\right] \operatorname{arctg} \frac{E}{2 Z \Omega_{1}},
$$

где $T_{\mathrm{c0}}$ - температура сверхпроводящего перехода в чистой неадиабатической системе, определяемая выражением

$$
T_{\mathrm{c} 0}=\frac{2 \omega_{0} \gamma}{\sqrt{e}(1+m) \pi} \exp \left(\frac{1}{2} \frac{m}{m+1}\right) \exp \left(-\frac{Z}{\lambda_{\triangle}}\right) .
$$


При этом выполнив суммирование по $\Omega_{1}$ в определении $Z(35)$, получаем

$$
Z=1+\lambda_{z} \frac{1}{1+m}, \quad m=\frac{2 \omega_{0}}{E} .
$$

Вычислив далее $F\left(\Omega_{1}\right)$ на основании (37) и (34) и подставив результат в $(43)$, получаем

$$
\ln \frac{T_{\mathrm{c}}}{T_{\mathrm{c} 0}}=\frac{2}{\beta} Z \sum_{\Omega_{1}} \frac{\omega_{0}^{4}}{\left(\Omega_{1}^{2}+\omega_{0}^{2}\right)^{2}}\left[\frac{Z \Omega_{1}+\tilde{a}+\tilde{b}-i I}{\left(Z \Omega_{1}+\tilde{a}\right)^{2}-\tilde{b}^{2}+I^{2}}-\frac{1}{Z \Omega_{1}}\right] \operatorname{arctg} \frac{E}{2 Z \Omega_{1}},
$$

где

$$
\begin{gathered}
\tilde{a}=\bar{a} \frac{2}{\pi} \operatorname{arctg} \frac{E}{2 \Omega Z}, \quad \tilde{b}=\bar{b} \frac{2}{\pi} \operatorname{arctg} \frac{E}{2 \Omega Z}, \\
\bar{a}=2 \bar{\Gamma}_{\mathrm{ex}}\left(\left\langle S_{x}^{2}\right\rangle+\left\langle S_{y}^{2}\right\rangle-2\left\langle S_{z}^{2}\right\rangle\right), \quad \bar{b}=-\Gamma_{\mathrm{ex}}\left(\left\langle S_{x}^{2}\right\rangle+\left\langle S_{y}^{2}\right\rangle\right) .
\end{gathered}
$$

Выполним в уравнении (46) ряд преобразований аналогично тому, как это было сделано в случае парамагнитной фазы [34]. В результате получаем

$$
\ln \frac{T_{\mathrm{c}}}{T_{\mathrm{c} 0}}=\Psi\left(\frac{1}{2}\right)-\frac{1}{2}\left[1-\frac{\bar{b}}{\sqrt{\bar{b}^{2}-I^{2}}}\right] \Psi\left(\frac{1}{2}+\rho_{+}\right)-\frac{1}{2}\left[1+\frac{\bar{b}}{\sqrt{\bar{b}^{2}-I^{2}}}\right] \Psi\left(\frac{1}{2}+\rho_{-}\right)
$$

где $\Psi$ - пси-функция Эйлера,

$$
\rho_{ \pm}=\frac{1}{Z} \frac{\bar{a}+\sqrt{\bar{b}^{2}-I^{2}}}{2 \pi T_{\mathrm{c}}} .
$$

Это уравнение описывает температуру сверхпроводящего перехода в фазе ферромагнитного упорядочения спинов атомов примеси и учитывает как парамагнитный эффект внутреннего поля, так и влияние процессов рассеяния электронов на примеси. По виду оно совпадает с соответствуюшим уравнением адиабатической теории [17], [35]. При этом происходит существенное переопределение величин $T_{\mathrm{c} 0}$ и $\rho_{ \pm}$. Величина $T_{\mathrm{c} 0}$ возрастает благодаря эффектам неадиабатичности и может достигать значений, присуших ВТСП-материалам при промежуточных значениях константы связи $\lambda$ [30], а параметры рассеяния $\bar{a}$ и $|\bar{b}|$ уменьшаются по сравнению со случаем адиабатических систем. В предельном случае $I=0$ (парамагнитная фаза) [34] решение уравнения (48) показывает замедленное убывание величины $T_{\mathrm{c}}$ с ростом концентрации примеси по сравнению со случаем обычных сверхпроводников и существенно зависит от параметра Мигдала $m=2 \omega_{0} / E$.

Очень важную роль в сосушествовании сверхпроводимости и ферромагнетизма в системе с тяжелыми компонентами играет спин-орбитальное взаимодействие [17]. Это взаимодействие способствует переворачиванию спинов электронов и смешиванию электронных состояний с противоположными спинами, ослабляя эффект "раздвижки” поверхности Ферми. Учет этого взаимодействия в неадиабатических системах приводит к переопределению величин $\bar{a}$ и $\bar{b}$. В этом случае имеем

$$
\bar{a}=\frac{2}{3} \bar{\Gamma}_{\mathrm{so}}+\bar{\Gamma}_{\mathrm{ex}} S(S+1)+\bar{\Gamma}_{\mathrm{ex}}\left\langle S_{z}^{2}\right\rangle, \quad \bar{b}=\frac{2}{3} \bar{\Gamma}_{\mathrm{so}}-\left(\left\langle S_{x}^{2}\right\rangle+\left\langle S_{y}^{2}\right\rangle\right)
$$


где $\bar{\Gamma}_{\text {so }}$ - затухание, связанное со спин-орбитальным рассеянием,

$$
\bar{\Gamma}_{\mathrm{so}}=\Gamma_{\mathrm{so}}\left[1+2 \lambda P_{V}\left(Q_{c}, 0,0\right)\right], \quad \Gamma_{\mathrm{so}}=\pi N_{0} c_{\mathrm{so}} V_{\mathrm{so}}^{2},
$$

$c_{\mathrm{so}}-$ концентрация немагнитной примеси, $V_{\mathrm{so}}$ - потенциал примесного рассеяния. Нетрудно видеть (см. (48), (50)), что при $I=0$ (парамагнитная фаза) спин-орбитальное взаимодействие не влияет на величину $T_{\mathrm{c}}$, а оказывает сушественное влияние только в ферромагнитной фазе $(I \neq 0)$.

\section{4. КРИТИЧЕСКАЯ КОНЦЕНТРАЦИЯ ПРИМЕСИ И ЗНАЧЕНИЯ $T_{\mathrm{C}}$ ВБЛИЗИ ТЕМПЕРАТУРЫ ФЕРРОМАГНИТНОГО УПОРЯДОЧЕНИЯ}

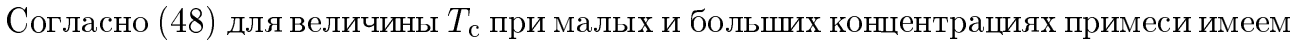
соответственно

$$
\begin{aligned}
\frac{T_{\mathrm{c}}}{T_{\mathrm{c} 0}}= & -\frac{\pi^{2}}{4}\left[1-\frac{\bar{b}}{\sqrt{\bar{b}^{2}-I^{2}}}\right] \rho_{+}-\frac{\pi^{2}}{4}\left[1+\frac{\bar{b}}{\sqrt{\bar{b}^{2}-I^{2}}}\right] \rho_{-}, \quad \rho_{ \pm} \ll 1 \\
T_{\mathrm{c}}^{2}= & \frac{6}{\pi^{2}} \frac{\left(\bar{a}^{2}-\bar{b}^{2}+I^{2}\right)^{2}}{(\bar{a}+\bar{b})^{2}-I^{2}} \times \\
& \times\left[\ln \left(\frac{\pi T_{\mathrm{c} 0}}{2 \gamma} \frac{1}{\sqrt{\bar{a}^{2}-\bar{b}^{2}+I^{2}}}\right)+\frac{\bar{b}}{2 \sqrt{\bar{b}^{2}-I^{2}}} \ln \frac{\bar{a}+\sqrt{\bar{b}^{2}-I^{2}}}{\bar{a}-\sqrt{\bar{b}^{2}-I^{2}}}\right], \quad \rho_{ \pm} \gg 1 .
\end{aligned}
$$

Полагая во второй формуле (51) $T_{\mathrm{c}}=0$, получаем выражение для критической концентрации примеси

$$
\ln \frac{\Gamma_{\mathrm{cr}}}{\Gamma_{\mathrm{cr}}^{\mathrm{p}}}=\ln \frac{2 \bar{\Gamma}}{\sqrt{\bar{a}^{2}-\bar{b}^{2}+I^{2}}}+\frac{\bar{b}}{2 \sqrt{\bar{b}^{2}-I^{2}}} \ln \frac{\bar{a}+\sqrt{\bar{b}^{2}-I^{2}}}{\bar{a}-\sqrt{\bar{b}^{2}-I^{2}}},
$$

где $\bar{\Gamma}=\bar{\Gamma}_{\text {ех }} S(S+1), \Gamma_{\text {сr }}^{\mathrm{p}}-$ критическая концентрация примеси в парамагнитной фазе, определяемая соотношением

$$
\Gamma_{\mathrm{cr}}^{\mathrm{p}}=\frac{\pi T_{\mathrm{c} 0}}{4 \gamma f_{c}}, \quad f_{c}=f_{c}(m)=\frac{1}{Z}\left[1-2 \lambda \frac{m}{1+m}\right]
$$

Последняя формула демонстрирует сушественное увеличение критической концентрации примеси в неадиабатических системах при $I=0$ по сравнению со случаем обычных сверхпроводников благодаря большим значениям $T_{\mathrm{c} 0}$ и $f_{c}(m)<1$. Температура сверхпроводяшего перехода $T_{\mathrm{c}}$ в ферромагнитной фазе определяется уравнением (48) или (51), а критическая концентрация примеси - соотношением (52). Однако получение явной зависимости $T_{\text {с }}$ от концентрации примеси - очень сложная задача, поскольку необходимо знать температурную зависимость величин $\left\langle S_{z}\right\rangle$ и $\left\langle S_{z}^{2}\right\rangle$. Для получения качес-

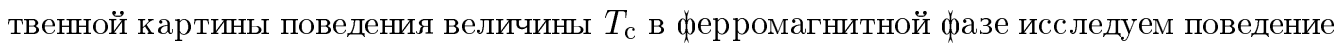
этой величины вблизи температуры магнитного упорядочения $T_{\mathrm{K}}$, определим наклон 
кривой $T_{\mathrm{c}}(\Gamma)$ и значения критических концентраций примеси в двух предельных случаяx $\Gamma_{\mathrm{so}} / \Gamma \gg 1$ и $\Gamma_{\mathrm{so}} / \Gamma \ll 1$. Для выполнения этой программы используем методику, применяемую при исследовании адиабатических систем [35], [36]. Исходим из факта,

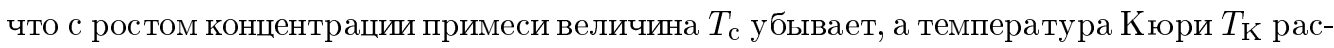
тет [17], [35]. Через $T^{*}$ обозначим точку пересечения этих двух величин, а через $c^{*}-$ соответствующую концентрацию примеси. На основании определения $T_{\mathrm{K}}$ в нормальном состоянии и уравнения для $T_{\mathrm{c}}$ в парамагнитной фазе получаем

$$
\begin{gathered}
T_{\mathrm{K}}=\frac{2}{3} c^{*} S(S+1) V_{2}^{2} N_{0} \\
\ln \frac{T^{*}}{T_{\mathrm{c} 0}}=\Psi\left(\frac{1}{2}\right)-\Psi\left(\frac{1}{2}+\frac{\bar{\Gamma}^{*}}{\pi \bar{T}^{*} Z}\right)=\Psi\left(\frac{1}{2}\right)-\Psi\left(\frac{1}{2}+\frac{3}{2} f_{c}\right) .
\end{gathered}
$$

При $m=0, f_{c}=1, T_{\mathrm{c0}}=T_{\mathrm{c0}}^{0}$ последняя из формул (54) переходит в соответствующее выражение адиабатической теории [17] для случая изотропного рассеяния примеси. Так как в нашем случае $m \neq 0, f_{c}(m)<1$ и $T_{\mathrm{c} 0}^{0}<T_{\mathrm{c} 0}$, то значение $T^{*}$ значительно превышает аналогичное значение в адиабатической теории. Исследуем поведение $T_{\mathrm{c}}$ вблизи значения $c=c^{*}$. С этой целью выполним разложение в уравнении (48) по величине $I^{2}$. В результате получим

$$
\begin{aligned}
\ln \frac{T_{\mathrm{c}}}{T_{\mathrm{c} 0}}= & \Psi\left(\frac{1}{2}\right)-\Psi\left(\frac{1}{2}+\frac{\bar{\Gamma}}{\pi T_{\mathrm{c}} Z}\right)-\frac{3 I^{2}}{8\left(\bar{\Gamma}_{\mathrm{so}}-\bar{\Gamma}\right)} \times \\
& \times\left\{\frac{3}{2\left(\bar{\Gamma}_{\mathrm{so}}-\bar{\Gamma}\right)}\left[\Psi\left(\frac{1}{2}+\frac{\bar{\Gamma}}{\pi T_{\mathrm{c}} Z}\right)-\Psi\left(\frac{1}{2}+\frac{2}{3} \bar{\Gamma}_{\mathrm{so}}+\frac{1}{3} \bar{\Gamma}\right)\right]+\right. \\
& \left.+\frac{1}{Z \pi T_{\mathrm{c}}} \Psi^{\prime}\left(\frac{1}{2}+\frac{\bar{\Gamma}}{\pi T_{\mathrm{c}} Z}\right)\right\}
\end{aligned}
$$

Разлагая далее это выражение по величинам $\left(T_{\mathrm{c}}-T^{*}\right) / T^{*}$ и $\left(\Gamma-\Gamma^{*}\right) / \Gamma^{*}$ с учетом условия $I\left(c^{*} T^{*}\right)=0$, получаем

$$
\frac{T_{\mathrm{c}}-T^{*}}{T^{*}}=\frac{Y}{Y-1} \frac{\bar{\Gamma}-\bar{\Gamma}^{*}}{\bar{\Gamma}^{*}}=\frac{Y}{Y-1} \frac{\Gamma-\Gamma^{*}}{\Gamma^{*}},
$$

где

$$
\begin{aligned}
Y=\frac{3 f_{c}}{2} & \Psi^{\prime}\left(\frac{1+3 f_{c}}{2}\right)-\frac{15}{2 f_{c}} \frac{1}{(S+1)^{2}+S^{2}}\left(\frac{1}{2 \pi N_{0} V_{2}}\right)^{2} \times \\
\times & \left(1-\frac{\bar{\Gamma}_{\mathrm{so}}}{\bar{\Gamma}^{*}}\right)^{-1}\left\{\Psi^{\prime}\left(\frac{1+3 f_{c}}{2}\right)+\frac{1}{f_{c}}\left(1-\frac{\bar{\Gamma}_{\mathrm{so}}}{\bar{\Gamma}^{*}}\right)^{-1} \times\right. \\
& \left.\times\left[\Psi\left(\frac{1}{2}+\frac{f_{c}}{2}\left(1+\frac{2 \bar{\Gamma}_{\mathrm{so}}}{\bar{\Gamma}^{*}}\right)\right)-\Psi\left(\frac{1+3 f_{c}}{2}\right)\right]\right\} .
\end{aligned}
$$

Значение величины $T_{\mathrm{c}}$ в ферромагнитной фазе вблизи $c^{*}$ зависит от параметров $\Gamma_{\mathrm{so}} / \Gamma, V_{2}, N_{0}$ и $f_{c}(m)$. Эффекты неадиабатичности в этих формулах проявляются в 
величине $f_{c}(m)<1$ при отличном от нуля значении параметра Мигдала $(m \neq 0)$. При $m=0, f_{c}(m)=1$ имеем результаты адиабатической теории для случая изотропного рассеяния примеси.

Рассмотрим два предельных случая: сильное спин-орбитальное взаимодействие $\left(\Gamma_{\mathrm{so}} / \Gamma \gg 1\right)$ и слабое спин-орбитальное взаимодействие $\left(\Gamma_{\text {so }} / \Gamma \ll 1\right)$. В этих двух предельных случаях получаем соответственно

$$
\begin{aligned}
Y= & \left\{\frac{3 f_{c}}{2}+\frac{15 S(S+1)}{2 f_{c}\left[(S+1)^{2}+S^{2}\right]}\left(\frac{1}{2 \pi N_{0} V_{2}}\right)^{2} \frac{\Gamma}{\Gamma_{\mathrm{so}}}\right\} \Psi^{\prime}\left(\frac{1+3 f_{c}}{2}\right), \\
Y= & \frac{3 f_{c}}{2} \Psi^{\prime}\left(\frac{1+3 f_{c}}{2}\right)-\frac{15}{2 f_{c}} \frac{1}{(S+1)^{2}+S^{2}}\left(\frac{1}{2 \pi N_{0} V_{2}}\right)^{2} \times \\
& \times\left\{\Psi^{\prime}\left(\frac{1+3 f_{c}}{2}\right)+\frac{1}{f_{c}}\left[\Psi\left(\frac{1}{2}+\frac{f_{c}}{2}\right)-\Psi\left(\frac{1}{2}+\frac{3 f_{c}}{2}\right)\right]\right\} .
\end{aligned}
$$

Из выражения (52) можно получить значение для критических концентраций примеси в ферромагнитной фазе в двух предельных случаях: $\bar{\Gamma}_{\text {so }} \ll \bar{\Gamma}$ и $\bar{\Gamma}_{\text {so }} \gg \bar{\Gamma}$. В первом случае с точностью до членов, линейных по $\Gamma_{\mathrm{so}} / \Gamma_{\mathrm{cr}}$, имеем

$$
\begin{aligned}
\ln \frac{\Gamma_{\mathrm{cr}}}{\Gamma_{\mathrm{cr}}^{\mathrm{p}}}= & \ln \frac{S+1}{\sqrt{S(S+1)+1 / \kappa^{2}}}-\frac{1}{4 \sqrt{1 / 4-1 / \kappa^{2}}} \ln \frac{S+1 / 2+\sqrt{1 / 4-1 / \kappa^{2}}}{S+1 / 2-\sqrt{1 / 4-1 / \kappa^{2}}}- \\
& -\frac{\Gamma_{\mathrm{so}}}{3 \Gamma_{\mathrm{cr}}}\left\{\frac{S+1}{S(S+1)+1 / \kappa^{2}}+\frac{1}{2 \kappa^{2} S \zeta^{3 / 2}} \ln \frac{S+1 / 2+\zeta}{S+1 / 2-\zeta}-\right. \\
& \left.-\frac{1}{4 S \zeta}\left[\frac{1 / 2+\zeta}{S+1 / 2-\zeta}+\frac{1 / 2-\zeta}{S+1 / 2+\zeta}\right]\right\}
\end{aligned}
$$

где

$$
\kappa=2 \pi N_{0} V_{2} f_{c}(m) Z, \quad \zeta=\sqrt{\frac{1}{4}-\frac{1}{\kappa^{2}}} .
$$

В соответствии с (61) параметр взаимодействия $\kappa$ уменьшается благодаря эффектам неадиабатичности, поскольку при $m \neq 0 \quad f_{c}(m)<1$. При $\kappa \ll 1$ выражение $(60)$ можно привести к виду

$$
\begin{aligned}
\ln \frac{\Gamma_{\mathrm{cr}}}{\Gamma_{\mathrm{cr}}^{\mathrm{p}}} \approx & \ln \frac{S+1}{\sqrt{S(S+1)+1 / \kappa^{2}}}-\frac{\kappa}{2} \operatorname{arctg} \frac{1}{\kappa(S+1 / 2)}- \\
& -\frac{\Gamma_{\mathrm{so}}}{3 \Gamma_{\mathrm{cr}}}\left\{\frac{S+1}{S(S+1)+1 / \kappa^{2}}-\kappa \operatorname{arctg} \frac{1}{\kappa(S+1 / 2)}+\right. \\
& \left.+\frac{\kappa^{2}}{4 S} \frac{S+1 / 2-2 / \kappa^{2}}{(S+1 / 2)^{2}+1 / \kappa^{2}}\right\} .
\end{aligned}
$$

В случае больших значений спин-орбитального взаимодействия $\bar{\Gamma}_{\text {so }} \gg \bar{\Gamma}$ из формулы (52) получаем

$$
\ln \frac{\Gamma_{\mathrm{cr}}}{\Gamma_{\mathrm{cr}}^{\mathrm{p}}} \approx-\frac{3 \bar{\Gamma}_{\mathrm{cr}}}{2 \bar{\Gamma}_{\mathrm{so}} \kappa^{2}(S+1)^{2}}\left[1+\frac{\bar{\Gamma}_{\mathrm{cr}}}{2 \bar{\Gamma}_{\mathrm{so}} / 3} \ln \frac{\bar{\Gamma}_{\mathrm{cr}}}{2 \bar{\Gamma}_{\mathrm{so}} / 3}\right] .
$$

Имеем $\Gamma_{\mathrm{cr}}<\Gamma_{\mathrm{cr}}^{\mathrm{p}}$, а с ростом $\Gamma_{\mathrm{so}}$ критическая концентрация $\Gamma_{\mathrm{cr}}$ совпадает с критической концентрацией в парамагнитной фазе. 


\section{5. ТЕМПЕРАТУРА ФЕРРОМАГНИТНОГО УПОРЯДОЧЕНИЯ}

Вычислим спиновую плотность электрона $\left\langle\sigma_{z}\right\rangle$, определяемую соотношением:

$$
\left\langle\sigma_{z}\right\rangle=\frac{1}{\beta V} \sum_{\vec{p}, \Omega}\left[\bar{G}_{\uparrow \uparrow}(\vec{p}, \Omega)-\bar{G}_{\downarrow \downarrow}(\vec{p}, \Omega)\right],
$$

где $\bar{G}$ - полная функция Грина, усредненная по положениям хаотически распределенной примеси и ориентациям спинов (29). В приближении слабой связи $\left(\Sigma_{1 \pm} / E \ll 1\right.$, $\left.\Sigma_{2 \pm} / E \ll 1\right)$ и небольших концентраций примеси $\left(\Gamma_{\mathrm{ex}} / E \ll 1\right)$ после интегрирования по энергии для величины $\left\langle\sigma_{z}\right\rangle$ получаем

$$
\begin{aligned}
& \left\langle\sigma_{z}\right\rangle-\left\langle\sigma_{z}\right\rangle_{n}=-N_{0} i \pi \sum_{\Omega}\left[\frac{1}{\sqrt{1+U_{1+}^{-2}+U_{2+}^{-2}}}-\frac{1}{\sqrt{1+U_{1-}^{-2}+U_{2-}^{-2}}}\right] \varphi(\Omega), \\
& \left\langle\sigma_{z}\right\rangle_{n}=-\frac{2 N_{0} I}{Z}, \quad U_{1 \pm}=\frac{\widetilde{\Sigma}_{1 \pm}}{\widetilde{\Omega}_{ \pm}}, \quad U_{2 \pm}=\frac{\widetilde{\Sigma}_{2 \pm}}{\widetilde{\Omega}_{ \pm}}, \quad \varphi(\Omega)=\frac{2}{\pi} \operatorname{arctg} \frac{E}{2 Z \Omega} .
\end{aligned}
$$

На основании соотношений $(30)$ для величин $U_{1 \pm}$ и $U_{2 \pm}$ нетрудно получить систему уравнений

$$
\begin{aligned}
Z \omega \pm i I= & U_{1 \pm} \Sigma-2 \bar{\Gamma}_{\mathrm{ex}}\left\langle S_{z}^{2}\right\rangle \frac{\varphi(\Omega)}{\sqrt{1+U_{1 \pm}^{-2}+U_{2 \pm}^{-2}}} \varphi(\Omega)+\frac{2}{3} \bar{\Gamma}_{\mathrm{so}}\left(\frac{U_{1 \pm}}{U_{1 \mp}}-1\right) \times \\
& \times \frac{\varphi(\Omega)}{\sqrt{1+U_{1 \mp}^{-2}+U_{2 \mp}^{-2}}}-2 \bar{\Gamma}_{\mathrm{ex}}\left[\frac{U_{1 \pm}}{U_{1 \mp}}+1\right] \frac{\varphi(\Omega)}{\sqrt{1+U_{1 \mp}^{-2}+U_{2 \mp}^{-2}}} .
\end{aligned}
$$

Представим $U_{1 \pm}=\operatorname{Re} U_{1} \pm i \operatorname{Im} U_{1}, U_{2 \pm}=\operatorname{Re} U_{2} \pm i \operatorname{Im} U_{2}$ и рассмотрим температуру, близкую к температуре Кюри $T_{\mathrm{K}}$, при которой $\operatorname{Im} U_{1,2} \ll \operatorname{Re} U_{1,2} \quad\left(\left\langle S_{z}\right\rangle-\right.$ малая величина). Выполнив расчеты аналогично тому, как это было сделано в случае обычных сверхпроводников [35], на основании формул (65) и (66), приведем выражение для температуры Кюри $T_{\mathrm{K}}$ к виду

$$
T_{\mathrm{K}}=T_{\mathrm{K} 0} \frac{\chi_{s}}{\chi_{n}},
$$

где

$$
\begin{aligned}
\frac{\chi_{s}}{\chi_{n}}= & 1-\pi T Z \sum_{\Omega} \Sigma^{2} \varphi(\Omega)\left\{\left[\Sigma^{2}+\left(\Sigma_{1} \operatorname{Re} U_{1}\right)^{2}\right]^{3 / 2}+\frac{4}{3} \bar{\Gamma}_{\mathrm{so}} \varphi(\Omega) \times\right. \\
& \left.\times\left[\Sigma^{2}+\left(\Sigma_{1} \operatorname{Re} U_{1}\right)^{2}\right]-\frac{2}{3} \bar{\Gamma}_{\mathrm{ex}} S(S+1)\left[\Sigma^{2}+2\left(\Sigma_{1} \operatorname{Re} U_{1}\right)^{2}\right]\right\}^{-1},
\end{aligned}
$$

где $\Sigma^{2}=\Sigma_{1}^{2}+\Sigma_{2}^{2}$. При этом величина $\Sigma_{1} \operatorname{Re} U_{1}$ удовлетворяет уравнению

$$
\Sigma_{1} \operatorname{Re} U_{1}=\frac{Z \Omega}{1-2 \bar{\Gamma}_{\mathrm{ex}} S(S+1) \varphi(\Omega)\left[\Sigma^{2}+\left(\Sigma_{1} \operatorname{Re} U_{1}\right)^{2}\right]^{-1 / 2}} .
$$


В области малых конщентраций примеси $\left(\bar{\Gamma}_{\text {ex }} / \Sigma \ll 1, \bar{\Gamma}_{\text {so }} / \Sigma \ll 1\right)$ имеем

$$
T_{\mathrm{K}}=T_{\mathrm{K} 0}\left[\frac{\pi}{3} \frac{\Gamma_{\mathrm{so}}}{\Delta} f_{c}+\frac{\pi}{6 \Delta} \Gamma f_{c}\right]
$$

где $\Delta=\Sigma / Z$.

Рассмотрим поведение величины $T_{\mathrm{K}}$ вблизи температуры сверхпроводяшего перехода $T_{\mathrm{c}}$. В этом случае в знаменателях выражений (67) и (68) можно положить $\Sigma=0$. В приближении слабой связи $(\Delta / E \ll 1, \Gamma / E \ll 1)$ получаем

$$
\frac{\chi_{s}}{\chi_{n}}=1+Z\left(T_{\mathrm{K}}\right)
$$

где

$$
\begin{aligned}
Z\left(T_{\mathrm{K}}\right)= & \frac{3 \Delta^{2}}{8 \pi T_{\mathrm{K}}} \frac{1}{\Gamma f_{c}}\left[1-\frac{\Gamma_{\mathrm{so}}}{\Gamma}\right]^{-1}\left\{\Psi^{\prime}\left(\frac{1}{2}+\frac{\Gamma f_{c}}{\pi T}\right)+\right. \\
& \left.+\frac{3 \pi T_{\mathrm{K}}}{2 \Gamma f_{c}}\left[1-\frac{\Gamma_{\mathrm{so}}}{\Gamma}\right]^{-1}\left[-\Psi\left(\frac{1}{2}+\frac{\Gamma f_{c}}{\pi T_{\mathrm{K}}}\right)+\Psi\left(\frac{1}{2}+\frac{\Gamma f_{c}}{3 \pi T_{\mathrm{K}}}+\frac{2 \Gamma f_{c}}{3 \pi T_{\mathrm{K}}}\right)\right]\right\},
\end{aligned}
$$

а параметр порядка $\Delta$ удовлетворяет уравнению

$$
\ln \frac{T}{T_{\mathrm{c} 0}}=\frac{\pi}{\beta} \sum_{\Omega} \frac{\omega_{0}^{4}}{\left(\Omega^{2}+\omega_{0}^{2}\right)^{2}}\left[\frac{1}{\sqrt{\Delta^{2}+\left(\Delta U_{1}\right)^{2}}}-\frac{1}{\Omega}\right] \varphi(\Omega) .
$$

Проведя в последнем уравнении разложение по $\Delta^{2}$ с учетом соотношения (68), в приближении слабой связи и при выполнении условий $\Gamma / \omega_{0} \ll 1, \Gamma / E \ll 1$ получаем

$$
\ln \frac{T}{T_{\mathrm{c} 0}}=\Psi\left(\frac{1}{2}\right)-\Psi\left(\frac{1}{2}+\frac{\Gamma f_{c}}{\pi T}\right)-\frac{1}{2} b_{1}\left(\frac{\Gamma f_{c}}{\pi T}\right) \frac{\Delta^{2}}{(2 \pi T)^{2}}
$$

где

$$
b_{1}\left(\frac{\Gamma f_{c}}{\pi T}\right)=-\frac{1}{2} \Psi^{\prime \prime}\left(\frac{1}{2}+\frac{\Gamma f_{c}}{\pi T}\right)-\frac{\Gamma f_{c}}{\pi T} \frac{1}{6} \Psi^{\prime \prime \prime}\left(\frac{1}{2}+\frac{\Gamma f_{c}}{\pi T}\right) .
$$

Вблизи точки $\left(T^{*}, c^{*}\right)$ имеем

$$
\frac{\Delta^{2}}{\left(2 \pi T_{\mathrm{K}}\right)^{2}}=-\frac{2}{b_{1}\left(3 f_{c} / 2\right)}\left\{\frac{T_{\mathrm{K}}-T^{*}}{T^{*}}\left[1-\frac{3 f_{c}}{2} \Psi^{\prime}\left(\frac{1}{2}+\frac{3 f_{c}}{2}\right)\right]+\frac{\Gamma-\Gamma^{*}}{\Gamma^{*}} \frac{3 f_{c}}{2} \Psi^{\prime}\left(\frac{1}{2}+\frac{3 f_{c}}{2}\right)\right\} .
$$

На основании формул (67) и (75) для температуры Кюри вблизи точки пересечения с температурой сверхпроводяшего перехода получаем

где

$$
\frac{T_{\mathrm{K}}-T^{*}}{T^{*}}=\frac{1-\alpha\left(3 f_{c} / 2\right) \Psi^{\prime}\left(1 / 2+3 f_{c} / 2\right)}{1+\alpha\left[1-\left(3 f_{c} / 2\right) \Psi^{\prime}\left(1 / 2+3 f_{c} / 2\right)\right]} \frac{\Gamma-\Gamma^{*}}{\Gamma^{*}}
$$

$$
\begin{aligned}
\alpha= & \frac{2}{f_{c} b_{1}\left(3 f_{c} / 2\right)}\left[1-\frac{\Gamma_{\mathrm{so}}}{\Gamma^{*}}\right]^{-1}\left\{\Psi^{\prime}\left(\frac{1}{2}+\frac{3 f_{c}}{2}\right)+\frac{1}{f_{c}}\left[1-\frac{\Gamma_{\mathrm{so}}}{\Gamma^{*}}\right]^{-1} \times\right. \\
& \left.\times\left[-\Psi\left(\frac{1}{2}+\frac{3 f_{c}}{2}\right)+\Psi\left(\frac{1}{2}+\frac{f_{c}}{2}+\frac{\Gamma_{\mathrm{so}}}{\Gamma^{*}} f_{c}\right)\right]\right\} .
\end{aligned}
$$

Формула (76) позволяет определить $T_{\mathrm{K}}$ вблизи температуры сверхпроводящего перехода $T_{\mathrm{c}}$ и наклон кривой $\left(d T_{\mathrm{K}} / d \Gamma\right)$, а формула $(69)$ - значение $T_{\mathrm{K}}$ в области малых концентраций примеси. Следовательно, можно представить качественную картину поведения $T_{\mathrm{K}}$ как функции концентрации примеси в сверхпроводящей фазе. 


\section{6. ЧИСЛЕННЫЕ РЕШЕНИЯ И ВЫВОДЫ}

На рис. 1 и 2 приведены зависимости температуры сверхпроводящего перехода $T_{\mathrm{c}}$ при ферромагнитном упорядочении примеси и температуры Кюри $T_{\mathrm{K}}$ в сверхпроводящем состоянии системы при различных значениях параметра Мигдала $m=2 \omega_{0} / E$. При этом кривые $1-3$ соответствуют значениям $T_{\mathrm{c}}$, а кривые $1^{\prime}-3^{\prime}-$ значениям $T_{\mathrm{K}}$. Расчеты выполнены на основании формул, приведенных в разделах 4 и 5 . Использованы формулы (56) и (76), позволяющие определить значения $T_{\mathrm{c}}$ и $T_{\mathrm{K}}$, а также наклон кривых $T_{\mathrm{c}}(\Gamma)$ и $T_{\mathrm{K}}(\Gamma)$ вблизи точки $\left(T^{*}, \Gamma^{*}\right)$ на фазовой диаграмме. Использованы также уравнения (62) и (63), определяюшие критические конщентрации примеси, а также формула (69), позволяюшая определить поведение $T_{\mathrm{K}}$ при малых концентрациях примеси.

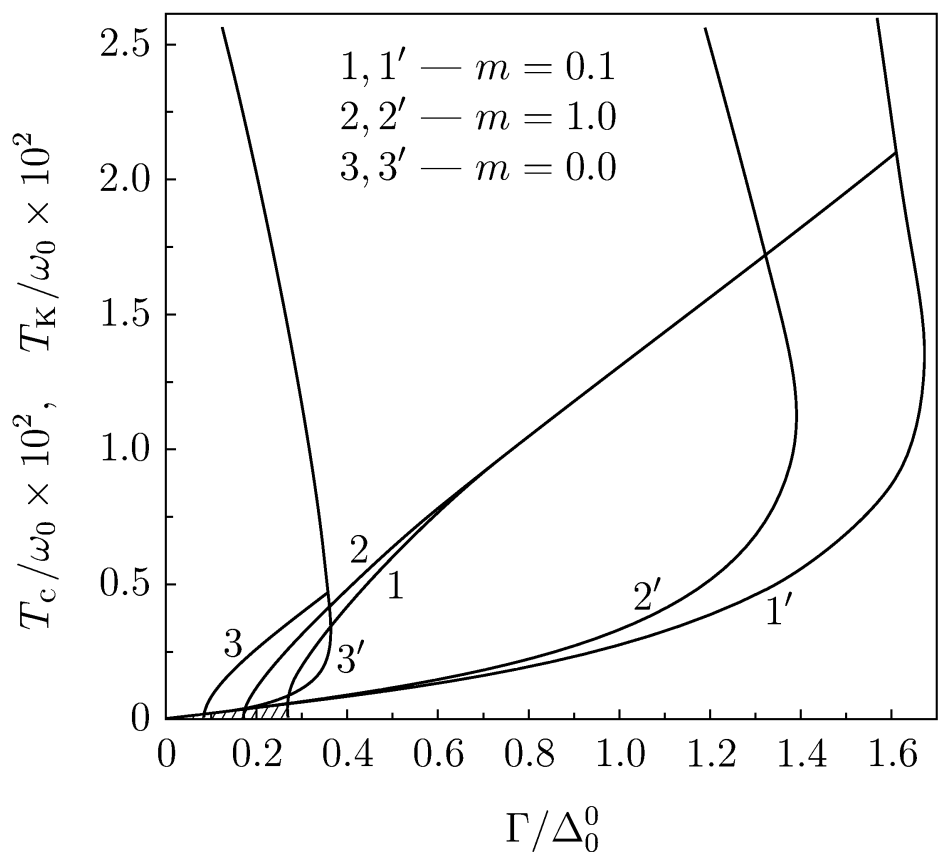

Рис. 1. Зависимость температуры сверхпроводящего перехода $T_{\mathrm{c}}$ и температуры Кюри $T_{\mathrm{K}}$ от концентрации примеси при $\Gamma_{\mathrm{So}}=0.1 \Gamma$. $\Delta_{0}^{0}$ - значение параметра порядка для чистого вещества адиабатической системы.

Рис. 1 отвечает случаю малых значений спин-орбитального взаимодействия $\left(\Gamma_{\mathrm{so}} / \Gamma \ll 1\right)$. Как следует из этого рисунка, в этом случае возможно сосуществование сверхпроводимости и ферромагнетизма в малой области низких температур и малых концентраций примеси. Величина этой области существенно зависит от параметра $m$. Она минимальна в адиабатических системах $(m=0)$, максимальна при $m=0.1$, затем уменьшается с ростом $m$, оставаясь больше области, присушей адиабатическим

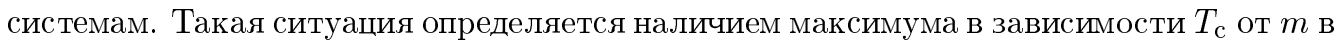
точке $m \approx 0.1$ [30]. На рис. 1 заштрихована область, соответствуюшая сосушествованию сверхпроводимости и ферромагнетизма: между кривыми $3^{\prime}$ и 3 мы имеем область, 
соответствуюшую адиабатическим системам $(m=0)$, между кривыми $2^{\prime}$ и $2-$ неадиабатическим системам $(m=1)$, между кривыми $1^{\prime}$ и 1 - случаю $m=0.1$.

Благодаря учету неадиабатичности область сосушествования сверхпроводимости и ферромагнетизма расширяется с ростом концентрации примеси. Имеет место также увеличение температуры $T^{*}$ и концентрации примеси $\Gamma^{*}$, при которых возникает ферромагнитное упорядочение примеси, в неадиабатических системах по сравнению с адиабатическими.

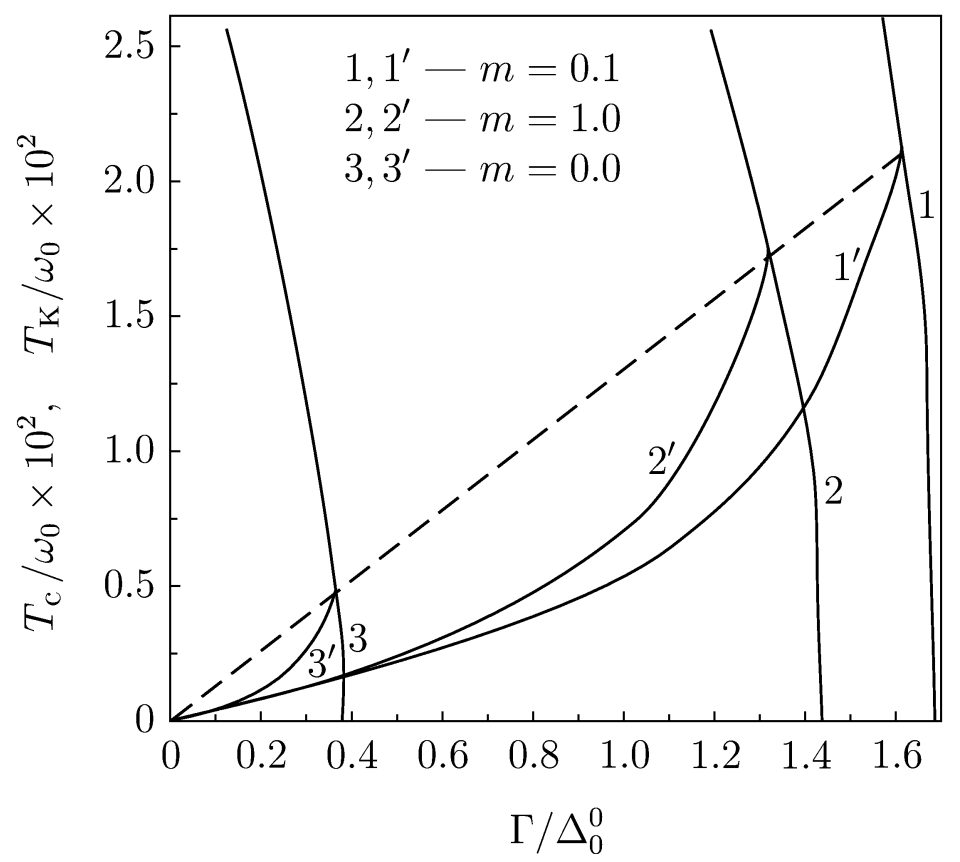

Рис. 2. То же, что на рис. 1 , но при $\Gamma_{\text {so }}=10 \Gamma$.

Рис. 2 отвечает случаю больших значений спин-орбитального взаимодействия $\left(\Gamma_{\mathrm{so}} / \Gamma \gg 1\right)$. В этом случае возникает более благоприятная ситуация для сосушествования сверхпроводимости и ферромагнетизма, поскольку наряду с обменным взаимодействием спин-орбитальное взаимодействие уменьшает "раздвижку" поверхности Ферми путем несохранения спина электрона. Величина $T_{\mathrm{c}}$ как функция концентрации примеси при заданных $m(m=0.1,1.0,0)$ переходит в соответствующие кривые парамагнитной фазы с критическими концентрациями $\Gamma_{\mathrm{cr}}=\Gamma_{\mathrm{cr}}^{\mathrm{p}}$, а $T_{\mathrm{K}}$ как функция концентрации примеси растет, имея вид вогнутой кривой. При этом между кривыми $T_{\mathrm{c}}$ и $T_{\mathrm{K}}$ возникает область сосушествования сверхпроводимости и ферромагнетизма. На рис. 2 для $m=0.1$ область сосушествования заключена между кривыми 1 и $1^{\prime}$, для $m=1$ - между кривыми 2 и $2^{\prime}$, для $m=0$ - между кривыми 3 и $3^{\prime}$. Таким образом, максимальная область сосушествования фаз соответствует значению $m=0.1$ и уменьшается c ростом $m$. Для любого $m$ из интервала $0.1<m<1$ эта область значительно больше, чем в случае адиабатических систем $(m=0)$. Величины $T^{*}$ и $\Gamma^{*}$ в неадиабатических 
системах при различных $m$ больше, чем в адиабатических.

На основании результатов исследования сверхпроводимости в системах с магнитной примесью можно сделать вывод, что эффекты неадиабатичности не только благоприятствуют сверхпроводимости в парамагнитной фазе [34], [37], [38], но и способствуют расширению области сосуществования сверхпроводимости и ферромагнетизма на фазовой диаграмме $(T, c)$.

\section{ПРИЛОЖКЕНИЕ}

Важным этапом в исследовании температуры сверхпроводяшего перехода неадиабатических систем с магнитной примесью является вычисление электрон-фононных и электрон-примесных вершинных и пересекающихся функций. Эти функции, определяемые соотношениями (14)-(16), содержат зависимость от частоты и обменного импульса, а также от концентрации магнитной примеси через внутреннее магнитное поле $I$ и параметр рассеяния $\rho_{ \pm}$. Для оценки этих величин мы обобщаем метод прямого вычисления, развитьй в работах [29], [30], на случай сверхпроводника с магнитно-упорядоченной примесью. С целью получения аналитических формул будем рассматривать приближение слабой связи $\left(T_{\mathrm{c}} \ll \omega_{0}, \quad I \ll \omega_{0}\right)$, а также небольшие концентрации примеси $\left(\Gamma_{1} / \omega_{0} \ll 1, \Gamma_{i} / \omega_{0} \ll 1\right)$. Используем выражение для функций Грина

$$
\bar{G}_{ \pm \pm}(\vec{p}, \Omega)=\left[i \widetilde{\Omega}_{ \pm}-\widetilde{\varepsilon}_{\vec{p} \pm}\right]^{-1}
$$

где

$$
\begin{gathered}
\widetilde{\Omega}_{ \pm}=\Omega \pm i I+\left(\Gamma_{1}+\Gamma_{\mathrm{ex}}\left[\left\langle S_{x}^{2}\right\rangle+\left\langle S_{y}^{2}\right\rangle+\left\langle S_{z}^{2}\right\rangle\right]\right) \operatorname{sgn} \Omega \\
\tilde{\varepsilon}_{\vec{p}_{ \pm}}=\varepsilon_{\vec{p}} \mp 4 i \Gamma_{12} \operatorname{sgn} \Omega .
\end{gathered}
$$

Эти выражения соответствуют адиабатической теории при $T=T_{\mathrm{c}}$. Использование выражения (П.1) для функции Грина обеспечивает первый порядок по неадиабатичности в определении массовых операторов (9), (10).

Расчеты выполняем в следующем порядке.

1. Произведение $\bar{G} \bar{G}$ соответствуюших функций Грина, входящих в определение функций $P_{V, c}(14)$, разложим на простые дроби [30], заменив суммирование по $\Omega$ интегрированием, как это делается при $T=0$. Это приближение оправдано рассмотрением слабой связи $\left(T_{\mathrm{c}} \ll \omega_{0}\right)$.

2. Рассмотрим квадратичный закон дисперсии энергии электронов и положим значение импульса обрезания электрон-фононного взаимодействия малым $\left(q_{c} \ll 2 p_{\mathrm{F}}\right)$. При таких значениях величины $q_{c}$ имеем $\vec{p} \approx \vec{p}_{1}$ и $\vec{p} \approx \vec{p}_{2}$, полагаем при этом $p \approx p_{\mathrm{F}}$. Такой подход позволяет так же, как и в [30], выразить значения $\varepsilon_{\vec{p}_{1}}+\vec{p}_{2}-\vec{p}$ И $\varepsilon_{\vec{p}_{2}}-\vec{p}_{1}-\vec{p}$ через $\varepsilon_{\vec{p}}$ и угловые зависимости. Получим

$$
\begin{aligned}
& \varepsilon_{\vec{p}_{2}+\vec{p}_{1}-\vec{p}} \approx \varepsilon_{\vec{p}_{2}}+E Q \alpha \cos \varphi \\
& \varepsilon_{\vec{p}_{2}-\vec{p}_{1}-\vec{p}} \approx \varepsilon_{\vec{p}_{2}}+E\left(1-Q^{2}\right) \frac{\alpha^{2}}{2}-E Q \sqrt{1-Q^{2}} \alpha \cos \varphi
\end{aligned}
$$

где $E=4 \varepsilon_{\mathrm{F}}, q=\left|\vec{p}-\vec{p}_{1}\right|, Q=q / 2 p_{\mathrm{F}}, \alpha$ - угол между $\vec{p}$ и $\vec{p}_{2}$. 
3. Выполним интегрирование по энергии $\varepsilon_{\vec{p}_{2}}$, полагая плотность электронных состояний $N(\varepsilon)=N_{0}$, а затем угловое интегрирование по $\alpha$ и $\varphi$ согласно соотношению

$$
\frac{1}{V} \sum_{\vec{p}_{1}} \ldots \rightarrow N_{0} \int_{0}^{2 \pi} \frac{d \varphi}{2 \pi} \int_{0}^{\pi} \frac{\sin \alpha d \alpha}{2} \int_{-E / 2}^{E / 2} \ldots d \varepsilon_{\vec{p}_{2}}
$$

4. Выполним усреднение по поверхности Ферми согласно формуле

$$
\begin{aligned}
P_{V, c}\left(Q_{c}, 0, \omega_{0}\right) & =\left(\frac{2 k_{\mathrm{F}}}{q_{c}}\right)^{2} \int \frac{d \Omega_{\vec{p}}}{4 \pi} \int \frac{d \Omega_{\vec{p}_{1}}}{4 \pi} \theta\left(q_{c}-\left|\vec{p}_{\mathrm{F}}-\vec{p}_{\mathrm{F} 1}\right|\right) P_{V, c}\left(\left|\vec{p}_{\mathrm{F}}-\vec{p}_{\mathrm{F} 1}\right|, 0, \omega_{0}\right)= \\
& =\frac{2}{Q_{c}^{2}} \int d Q Q P_{V, c}\left(Q, Q_{c}, 0, \omega_{0}\right), \quad Q_{c}=\frac{q_{c}}{2 p_{\mathrm{F}}}
\end{aligned}
$$

После выполнения этих операций получаем соотношения (32), где

$$
\begin{aligned}
& P_{V}^{0}\left(Q_{c}, 0, \omega_{0}\right)=-\varphi(m)+\left[\frac{\pi}{4}-\operatorname{arctg} \frac{m}{1+m}+\varphi(m)\right] \frac{m^{2}}{4 Q_{c}^{2}} \times \\
& \times\left[\sqrt{\eta_{c}(m)}-1-\ln \frac{1+\sqrt{\eta_{c}(m)}}{m}\right], \\
& P_{c}^{0}\left(Q_{c}, 0, \omega_{0}\right)=-\varphi(m)+\left[\frac{\pi}{4}-\operatorname{arctg} \frac{m}{1+m}+\varphi(m)\right] \frac{m}{4 Q_{c}^{2}\left(1-Q_{c}^{2} / 2\right)} \times \\
& \times \operatorname{arctg} \frac{4 Q_{c}^{2}\left(1-Q_{c}^{2} / 2\right)}{m}, \\
& \varphi(m)=m(1+m) \frac{(1+m)^{2}+2 m^{2}}{\left[(1+m)^{2}+m^{2}\right]^{2}}, \quad \eta_{c}(m)=1+\left(\frac{4 Q_{c}^{2}}{m}\right)^{2}, \\
& P_{V}\left(Q_{c}, 0,0\right)=-\frac{m}{m+1}, \quad m=\frac{2 \omega_{0}}{E} .
\end{aligned}
$$

Аналогичный способ вычислений используем и для примесных величин $R_{V, c}$ и $K_{V, c}$. Peзультаты этих расчетов в принятом вьше приближении приведены в разделе 3 (см (32)). Случай парамагнитной фазы $(I=0)$ рассмотрен нами в работе [34].

Благодарности. Автор выражает искреннюю признательность В. А. Москаленко за интерес к работе и обсуждение результатов, а Ф. Г. Кочорбэ за помошь в численных расчетах. Данное исследование финансируется грантом Высшего Совета по науке и технологическому развитию Молдовы. 


\section{Список литературы}

[1] O. Gunnarson. Rev. Mod. Phys. 1997. V. 69. P. 575.

[2] W. Ku, W. E. Pickett, R. T. Scalettar, A. G. Eguiluz. Ab initio investigation of collective charge excitations on $\mathrm{MgB}_{2}$. cond-mat/0105389.

[3] A. Lanzara, P. V. Bogdanov, X. I. Zhou et al. Nature. 2001. V. 412. P. 510.

[4] Y. Bardeen, Y. N. Cooper, Y. R. Shrieffer. Phys. Rev. 1957. V. 106. P. 162; 1957. V. 108. P. 1175.

[5] Н. Н. Боголюбов, Д. Н. Зубарев, Ю. А. Церковников. ДАН СССР. 1957. Т. 117. С. 788.

[6] Н. Н. Боголюбов. ЖЭТФ. 1958. Т. 34. С. 58; С. 73.

[7] Н. Н. Боголюбов, В. В. Толмачев, Д. В. Ширков. Новый метод в теории сверхпроводимости. М.: АН СССР, 1958.

[8] Д. Н. Зубарев. УФН. 1960. Т. 71. С. 71.

[9] А. В. Мигдал. ЖЭТФ. 1958. Т. 34. С. 1438.

[10] A. С. Элиашберг. ЖЭТФ. 1960. Т. 38. С. 966.

[11] W. L. McMillan. Phys. Rev. 1968. V. 167. P. 331.

[12] Н. Н. Боголюбов. УФН. 1959. Т. 67. С. 549.

[13] Л. П. Горьков. ЖЭТФ. 1958. Т. 34. С. 735.

[14] Л. А. Абрикосов, Л. П. Горьков. ЖЭТФ. 1958. Т. 35. С. 1558.

[15] А. А. Абрикосов, Л. П. Горьков. ЖЭТФ. 1960. Т. 39. С. 1781.

[16] S. Scalski, O. Betbeder-Matibet, P. R. Weiss. Phys. Rev. A. 1964. V. 136. P. 1500.

[17] Л. П. Горьков, А. И. Русинов. ЖЭТФ. 1964. Т. 46. С. 1363.

[18] Н. М. Плакида. Высокотемпературные сверхпроводники. М.: Международная программа образования, 1996.

[19] V. A. Moskalenko, P. Entil, D. F. Digor. Phys. Rev. B. 1999. V. 59. P. 619.

[20] W. Weber. Adv. Solid State Phys. 1988. V. 28. P. 141.

[21] В. А. Гинзбург, Е. Г. Максимов. СФХТ. 1992. Т. 5. С. 1453.

[22] G. M. Vujicic, V. L. Aksenov, N. M. Plakida, S. Stamenkovic. Phys. Lett. A. 1979. V. 73. P. 439; J. Phys. C. 1981. V. 14. P. 2377.

[23] N. M. Plakida, V. L. Aksenov, S. S. Dreschler. Europhys. Lett. 1987. V. 4. P. 1309.

[24] J. E. Hirsch, D. J. Scalapino. Phys. Rev. Lett. 1986. V. 56. P. 2732.

[25] J. Fridel. J. Phys.: Condens. Matter. 1989. V. 1. P. 7757.

[26] В. А. Москаленко, М. Е. Палистрант, В. М. Вакалюк. УФН. 1991. Т. 161. С. 155.

[27] Ф. Г. Кочорбә, М. Е. Палистрант. ЖЭТФ. 1993. Т. 104. С. 3084; ТМФ. 1993. Т. 96. C. 459.

[28] O. V. Danylenko, O. V. Dolgov. Phys. Rev. B. 2001. V. 63. P. 094506-1; cond-mat/0007189.

[29] L. Pietronero, S. Strässler, C. Grimaldi. Phys. Rev. B. 1995. V. 52. P. 10516.

[30] C. Grimaldi, L. Pietronero, S. Strässler. Phys. Rev. B. 1995. V. 52. P. 10530.

[31] M. L. Kulik, R. Zeyher. Phys. Rev. B. 1994. V. 49. P. 4395.

[32] R. Zeyher, M. L. Kulik. Phys. Rev. B. 1996. V. 53. P. 2850.

[33] А. А. Абрикосов, Л. П. Горьков, И. Е. Дзялошинский. Методы квантовой теории поля в статистической физике. М.: Наука, 1962.

[34] М. Е. Палистрант. ТМФ. 1999. Т. 119. С. 455.

[35] Yu. A. Izymov, Yu. N. Skryabin. Phys. Stat. Sol. (b). 1974. V. 61. P. 9.

[36] T. Tzuzuki. Progr. Theor. Phys. 1966. V. 35. P. 183.

[37] M. E. Palistrant, F. G. Kochorbe. J. Superconduct.: Incorp. Novel Magnetism. 2002. V. 15. P. 113.

[38] М. Е. Палистрант. ФНТ. 2002. Т. 28. С. 157. 